\title{
Evidence for natural hybridisation and novel Wolbachia strain superinfections in the Anopheles gambiae complex from Guinea
}

\author{
Claire L Jeffries ${ }^{1}$, Cintia Cansado-Utrilla ${ }^{1 \dagger}$, Abdoul H Beavogui ${ }^{2}$, Caleb Stica ${ }^{1}$, Eugene K \\ Lama $^{3}$, Mojca Kristan ${ }^{1}$, Seth R Irish ${ }^{4} \&$ Thomas Walker ${ }^{1} *$ \\ ${ }^{1}$ Department of Disease Control, Faculty of Infectious and Tropical Diseases, London School of Hygiene and Tropical \\ Medicine, London, WC1E 7HT, UK \\ ${ }^{2}$ Centre National de Formation et de Recherche en Santé Rurale de Mafèrinyah B.P. 2649, Conakry, Guinea \\ ${ }^{3}$ Programme National de Lutte contre le Paludisme, Guinée. B.P. 6339. Conakry, Guinea \\ ${ }^{4}$ The US President's Malaria Initiative and Entomology Branch, Centers for Disease Control and Prevention, Atlanta, \\ Georgia, 30329-4027, USA
}

Keywords: Wolbachia, mosquitoes, malaria, Anopheles, endosymbionts

\section{Summary}

Wolbachia, a widespread bacterium which can influence mosquito-borne pathogen transmission, has recently been detected within Anopheles (An.) species that are malaria vectors in Sub-Saharan Africa. Although studies have reported Wolbachia strains in the An. gambiae complex, apparent low density and prevalence rates require confirmation. In this study, wild Anopheles mosquitoes collected from two regions of Guinea were investigated. In contrast to previous studies, RNA was extracted from adult females $(n=516)$ to increase the chances for detection of actively expressed Wolbachia genes, determine Wolbachia prevalence rates and estimate relative strain densities. Molecular confirmation of mosquito species and Wolbachia Multilocus sequence typing (MLST) were carried out to analyse phylogenetic relationships of mosquito hosts and newly discovered Wolbachia strains. Strains were detected in An. gambiae s.s. (prevalence rates of 0.0-2.8\%) from the Faranah region, $A n$. melas (prevalence rate of $11.6 \%$ - 16/138) and hybrids between these two species (prevalence rate of $40.0 \%$ - 6/15) from Senguelen in the Maferinyah region. Furthermore, a novel high-density strain, termed wAnsX, was found in an unclassified Anopheles species. The discovery of novel Wolbachia strains (particularly in members, and hybrids, of the An. gambiae complex) provides further candidate strains that could be used for future Wolbachia-based malaria biocontrol strategies.

\section{Introduction}

Wolbachia endosymbiotic bacteria are estimated to infect $\sim 40 \%$ of insect species [1] and natural infections have been shown to have inhibitory effects on human arboviruses in mosquitoes [2-4]. High density Wolbachia strains have been utilised for mosquito biocontrol strategies targeting arboviruses as they induce synergistic phenotypic effects. Wolbachia strains that have been transinfected into Aedes (Ae.) aegypti and Ae. albopictus induce inhibitory effects on arboviruses, with maternal transmission and cytoplasmic incompatibility (CI) enabling introduced strains to spread through populations [5-13]. The successful release and establishment of

*Author for correspondence (Thomas.walker@1shtm.ac.uk).

$\dagger$ Present address: Departments of Vector Biology and Tropical Disease Biology, Centre for Neglected Tropical Diseases, Liverpool School of Tropical Medicine, Liverpool, UK 
bioRxiv preprint doi: https://doi.org/10.1101/772855; this version posted November $17,2020$. The copyright holder for this preprint (which was not certified by peer review) is the author/funder, who has granted bioRxiv a license to display the preprint in perpetuity. It is made available under aCC-BY-NC-ND 4.0 International license.

Wolbachia-transinfected Ae. aegypti populations in Cairns, Australia [14] was followed by further evidence of strong inhibitory effects on arboviruses from field populations [15]. Further studies in Australia [16, 17] and Kuala Lumpur, Malaysia [18] have now shown that Wolbachia frequencies have remained stable since initial releases and there is a reduction in human dengue incidence (case notifications) in the release sites.

The potential for Wolbachia to be used for biocontrol strategies targeting malaria transmission by Anopheles species has also been postulated [19] and initial laboratory experiments demonstrated that transient infections in An. gambiae reduce the density of Plasmodium (P.) falciparum parasites [20]. However, as with arboviruses there is variability in the level of inhibition of malaria parasites for different Wolbachia strains in different mosquito species [21-23]. A major step forward was achieved through the transinfection of a Wolbachia strain from Ae. albopictus (wAlbB) into An. stephensi and the confirmation of P. falciparum inhibition [24]. The interest in using Wolbachia for biocontrol strategies targeting malaria transmission in Anopheles mosquitoes has further increased due to the detection of natural strains of Wolbachia residing in numerous malaria vectors of Sub-Saharan Africa [25-29]. The An. gambiae complex, which consists of multiple morphologically indistinguishable species including several major malaria vector species, appears to contain diverse Wolbachia strains (collectively named wAnga) at both low prevalence and low infection densities [25, 26, 28-31]. In contrast, the recently discovered $w$ AnM and $w$ AnsA strains, found in $A n$. moucheti and An. species A respectively, are higher density infections that dominate the mosquito microbiome [26].

Interestingly, the presence of Wolbachia strains in Anopheles was inversely correlated to other bacteria species such as Asaia that are stably associated with several species [32-34]. Evidence for this 'mutual exclusion' between bacterial species in Anopheles was also present from analysis of field collected mosquitoes from multiple countries in Sub-Saharan Africa [26]. In this study, we collected wild Anopheles mosquitoes from two regions of Guinea in June-July 2018 and characterised the natural Wolbachia strains to provide further evidence for the presence of these endosymbionts in malaria vectors. In contrast to previous studies, we extracted RNA to make any detection of Wolbachia more likely to be from actively expressed Wolbachia genes and undertook qRT-PCR analysis to compare Wolbachia densities. Phylogenetic analysis revealed the presence of novel strains in An. melas, An. gambiae s.s.-melas hybrids (including Wolbachia superinfections within individual mosquitoes) and an unclassified Anopheles species.

\section{Materials and Methods}

Study sites \& collection methods. Anopheles adult mosquitoes were collected in 2018 from two regions (subprefectures) in Guinea; Faranah and Maferinyah. Human landing catches (HLCs) and larval dipping were conducted in three villages in the Faranah Prefecture; Balayani (10.1325, -10.7443), Foulaya (10.144633, 10.749717), and Tindo (9.9612230, -10.7016560) [35]. Three districts were selected for mosquito collections in the Maferinyah sub-prefecture using a variety of traps [36]. BG sentinel 2 traps (BG2) (Biogents), CDC light traps (John W. Hock), gravid traps (BioQuip) and stealth traps (John W. Hock) were used to sample adult mosquitoes in Maferinyah Centre I (09.54650, -013.28160), Senguelen (09.41150, -013.37564) and Fandie (09.53047, -013.24000). Mosquitoes collected from traps and HLCs were morphologically identified using keys and stored in RNAlater (Invitrogen) at $-70^{\circ} \mathrm{C}[35,36]$.

RNA extraction and generation of cDNA. RNA was extracted from individual whole female mosquitoes using Qiagen 96 RNeasy Kits according to manufacturer's instructions and a Qiagen Tissue Lyser II (Hilden, Germany) with a $5 \mathrm{~mm}$ stainless steel bead (Qiagen) to homogenise mosquitoes. RNA was eluted in $45 \mu \mathrm{L}$ of RNase-free water and stored at $-70^{\circ} \mathrm{C}$. RNA was reverse transcribed into complementary DNA (cDNA) using an Applied Biosystems High Capacity cDNA Reverse Transcription kit. A final volume of $20 \mu \mathrm{L}$ contained 10 $\mu \mathrm{L}$ RNA, $2 \mu \mathrm{L}$ 10X RT buffer, $0.8 \mu \mathrm{L} 25 \mathrm{X}$ dNTP $(100 \mathrm{mM}), 2 \mu \mathrm{L} 10 \mathrm{X}$ random primers, $1 \mu \mathrm{L}$ reverse transcriptase and $4.2 \mu \mathrm{L}$ nuclease-free water. Reverse transcription was undertaken in a Bio-Rad T100 Thermal Cycler as follows: $25^{\circ} \mathrm{C}$ for $10 \mathrm{~min}, 37^{\circ} \mathrm{C}$ for $120 \mathrm{~min}$ and $85^{\circ} \mathrm{C}$ for $5 \mathrm{~min}$ and cDNA stored at $-20^{\circ} \mathrm{C}$.

Molecular mosquito species identification. Species identification of the An. gambiae complex was initially undertaken using diagnostic species-specific PCR assays targeting the ribosomal intergenic spacer (IGS)[37] and SINE200 insertion[38] to distinguish between the morphologically indistinguishable sibling species. To confirm species identification for samples of interest and samples that could not be identified by species- 
specific PCR, Sanger sequencing and phylogenetic analysis was performed for PCR products from a range of gene targets including ribosomal IGS and internal transcribed spacer 2 (ITS2)[39] and mitochondrial cytochrome c oxidase subunit $1(\mathrm{COI})$ [40], cytochrome c oxidase subunit 2 (COII)[41] and NADH dehydrogenase subunits 4 and 5 (ND4-ND5)[42]. Where ITS2 PCR products for a particular sample were not successfully generated, or the sequencing generated was not of sufficient quality for onward analysis, a slight modification to the ITS2 primers was used to attempt to increase the success of amplification and sequencing. Alternative ITS2 primers adjusted from those published[39] were ITS2A-CJ: 5'-

TGTGAACTTGCAGGACACAT-3' and ITS2B-CJ: 5'-TATGCTTAAATTYAGGGGGT-3'. For confirmation of Culex $(C x$.) watti - a species collected in the same location and used for comparative Wolbachia density analysis - a different fragment of the mitochondrial cytochrome c oxidase subunit 1 (COI) gene [43] was sequenced given the lack of available sequences in certain regions for this species and to optimise sequencing quality and species discrimination. PCR reactions for IGS, SINE200, ITS2 and COI were prepared as previously described[36]. For COII amplification, PCR reactions were prepared using $10 \mu \mathrm{L}$ of Phire Hot Start II PCR Master Mix (Thermo Scientific ${ }^{\mathrm{TM}}$ ) with a final concentration of $1 \mu \mathrm{M}$ of each primer, $1 \mu \mathrm{L}$ of PCR grade water and $2 \mu \mathrm{L}$ template cDNA, to a final reaction volume of $20 \mu \mathrm{L}$. PCR reactions were carried out in a Bio-Rad T100 Thermal Cycler and cycling was $98^{\circ} \mathrm{C}$ for $30 \mathrm{sec}$ followed by 34 cycles of $98^{\circ} \mathrm{C}$ for $5 \mathrm{sec}, 55^{\circ} \mathrm{C}$ for $5 \mathrm{sec}$, $72^{\circ} \mathrm{C}$ for $30 \mathrm{sec}$ followed by $72^{\circ} \mathrm{C}$ for $1 \mathrm{~min}$. For ND4-ND5 PCR reactions were prepared using $10 \mu \mathrm{L}$ of HotStart Taq 2x Master Mix (New England BioLabs ${ }^{\circledR}$ ) with a final concentration of $2 \mu \mathrm{M}$ of each primer, $1 \mu \mathrm{L}$ of PCR grade water and $2 \mu \mathrm{L}$ template cDNA, to a final reaction volume of $20 \mu \mathrm{L}$. PCR reactions were carried out in a Bio-Rad T100 Thermal Cycler and cycling was $95^{\circ} \mathrm{C}$ for $30 \mathrm{sec}$ followed by 35 cycles of $95^{\circ} \mathrm{C}$ for $30 \mathrm{sec}$, $53^{\circ} \mathrm{C}$ for $60 \mathrm{sec}, 68^{\circ} \mathrm{C}$ for $90 \mathrm{sec}$ followed by $68^{\circ} \mathrm{C}$ for $5 \mathrm{~min}$. PCR products were separated and visualised using 2\% E-Gel EX agarose gels (Invitrogen) with SYBR safe and an Invitrogen E-Gel iBase Real-Time Transilluminator.

Wolbachia detection and amplification of Wolbachia genes. Wolbachia detection was first undertaken targeting the conserved Wolbachia genes previously shown to amplify a wide diversity of strains; $16 \mathrm{~S} r \mathrm{RNA}$ gene using primers W-Spec-16S-F: 5'-CATACCTATTCGAAGGGATA-3' and W-Spec-16s-R: 5'AGCTTCGAGTGAAACCAATTC-3 [44] and Wolbachia surface protein (wsp) gene using primers wsp81F: 5'TGGTCCAATAAGTGATGAAGAAAC-3' and wsp691R: 5'-AAAAATTAAACGCTACTCCA-3' [45]. PCR analysis was also undertaken on CDNA to determine if there was any evidence for the presence of CI-inducing genes CifA (primers 5'-TGTGGTAGGGAAGGAAAGAGGAAA-3', 5' -ATTCCAAGGACCATCACCTACAGA$3^{\prime}$ ) and CifB (primers 5'-TGCGAGAGATTAGAGGGCAAAATC-3', 5'-CCTAAGAAGGCTAATCTCAGACGC$3^{\prime}$ ) [46]. Multilocus strain typing (MLST) was undertaken to characterize Wolbachia strains using the sequences of five conserved genes as molecular markers to genotype each strain. In brief, 450-500 base pair fragments of the $g a t B, \operatorname{cox} A, h c p A, f t s Z$ and $f b p A$ Wolbachia genes were amplified from individual Wolbachia-infected mosquitoes using previously optimised protocols [47, 48]. Primers used were as follows: gatB_F1: 5' GAKTTAAAYCGYGCAGGBGTT-3', gatB_R1: 5'-TGGYAAYTCRGGYAAAGATGA-3', coxA_F1: 5' TTGGRGCRATYAACTTTATAG-3', coxA_R1: 5'-CTAAAGACTTTKACRCCAGT-3', hcpA_F1: 5' GAAATARCAGTTGCTGCAAA-3', hcpA_R1: 5'-GAAAGTYRAGCAAGYTCTG-3', ftsZ_F1: 5'ATYATGGARCATATAAARGATAG-3', ftsZ_R1: 5'-TCRAGYAATGGATTRGATAT-3', fbpA_F1: 5' GCTGCTCCRCTTGGYWTGAT-3' and fbpA_R1: 5'-CCRCCAGARAAAAYYACTATTC-3' with the addition of M13 adaptors. If no amplification was detected using standard primers, further PCR analysis was undertaken using degenerate primer sets, with or without M13 adaptors [47]. In selected An. melas specimens where Wolbachia $16 S$ rRNA Sanger sequencing (detailed below) indicated the possibility of superinfections, further MLST testing was carried out utilising Wolbachia Supergroup A and B strain specific primers [47]. PCR reactions were prepared using $10 \mu \mathrm{L}$ of Phire Hot Start II PCR Master Mix (Thermo Scientific ${ }^{\mathrm{TM}}$ ) with a final concentration of $1 \mu \mathrm{M}$ of each primer, $1 \mu \mathrm{L}$ of PCR grade water and $2 \mu \mathrm{L}$ template cDNA, to a final reaction volume of $20 \mu \mathrm{L}$. PCR reactions were carried out in a Bio-Rad T100 Thermal Cycler using variable optimised cycling conditions. For gat $B, h c p A$ and $f b p A$ genes cycling was $98^{\circ} \mathrm{C}$ for $30 \mathrm{sec}$ followed by 34 cycles of $98^{\circ} \mathrm{C}$ for $5 \mathrm{sec}, 65^{\circ} \mathrm{C}$ for $5 \mathrm{sec}, 72^{\circ} \mathrm{C}$ for $10 \mathrm{sec}$ followed by $72^{\circ} \mathrm{C}$ for $1 \mathrm{~min}$. For cox $A$ and ftsZ genes cycling was $98^{\circ} \mathrm{C}$ for $30 \mathrm{sec}$ followed by 34 cycles of $98^{\circ} \mathrm{C}$ for $5 \mathrm{sec}, 55^{\circ} \mathrm{C}$ for $5 \mathrm{sec}, 72^{\circ} \mathrm{C}$ for $30 \mathrm{sec}$ followed by $72^{\circ} \mathrm{C}$ for 1 min. PCR products were separated and visualised using 2\% E-Gel EX agarose gels (Invitrogen) with SYBR safe and an Invitrogen E-Gel iBase Real-Time Transilluminator.

Sanger sequencing. PCR products were submitted to Source BioScience (Source BioScience Plc, Nottingham, UK) for PCR reaction clean-up, followed by Sanger sequencing to generate both forward and 
bioRxiv preprint doi: https://doi.org/10.1101/772855; this version posted November $17,2020$. The copyright holder for this preprint (which was not certified by peer review) is the author/funder, who has granted bioRxiv a license to display the preprint in perpetuity. It is made available under aCC-BY-NC-ND 4.0 International license.

reverse reads. Where Wolbachia PCR primers included M13 adaptors, just the M13 primers alone

(M13_adaptor_F: 5'-TGTAAAACGACGGCCAGT-3' and M13_adaptor_R: 5'-CAGGAAACAGCTATGACC-

$3^{\prime}$ ) were used for sequencing, otherwise the same primers as utilised for PCR were used. Sequencing analysis was carried out in MEGAX [49]. Both chromatograms (forward and reverse traces) from each sample were manually checked, edited, and trimmed as required, followed by alignment by ClustalW and checking to produce consensus sequences. Consensus sequences were used to perform nucleotide BLAST (NCBI) database queries, and for Wolbachia genes searches against the Wolbachia MLST database (http://pubmlst.org/Wolbachia). If a sequence produced an exact match in the MLST database we assigned the appropriate allele number, otherwise we obtained a new allele number for each novel gene locus sequence for Anopheles Wolbachia strains through submission of the FASTA and raw trace files on the Wolbachia MLST website for new allele assignment and inclusion within the database. Full consensus sequences were also submitted to GenBank and assigned accession numbers. The Sanger sequencing traces from the wsp gene were also treated in the same way and analysed alongside the MLST gene locus scheme, as an additional marker for strain typing. Where potential mixed strains were detected (in An. melas and An. gambiae s.s-melas hybrid individuals) and any further Supergroup A or B specific testing was exhausted, it wasn't possible to submit these sequences to the MLST database for a new allele to be assigned, however, clean $16 \mathrm{~S}$ consensus sequences from representative individuals for each of the Supergroup A and B strains characterised were submitted to GenBank, in addition to the full MLST profile of one individual demonstrating one of the Supergroup A strain infections.

Phylogenetic analysis. Alignments were constructed in MEGAX by ClustalW to include all relevant and available sequences highlighted through searches on the BLAST and Wolbachia MLST databases. Maximum Likelihood phylogenetic trees were constructed from Sanger sequences as follows. The evolutionary history was inferred by using the Maximum Likelihood method based on the Tamura-Nei model [50]. The tree with the highest log likelihood in each case is shown. The percentage of trees in which the associated taxa clustered together is shown next to the branches. Initial tree(s) for the heuristic search were obtained automatically by applying Neighbor-Join and BioNJ algorithms to a matrix of pairwise distances estimated using the Maximum Composite Likelihood (MCL) approach, and then selecting the topology with superior log likelihood value. The trees are drawn to scale, with branch lengths measured in the number of substitutions per site. Codon positions included were $1 \mathrm{st}+2 \mathrm{nd}+3 \mathrm{rd}+$ Noncoding. All positions containing gaps and missing data were eliminated. The phylogeny test was by Bootstrap method with 1000 replications. Evolutionary analyses were conducted in MEGAX [49].

Wolbachia quantification. To estimate Wolbachia density across multiple mosquito species, RNA extracts were added to Qubit TM RNA High Sensitivity Assays (Invitrogen) and total RNA measured using a Qubit 4 Fluorometer (Invitrogen). All RNA extracts were then diluted to produce extracts that were 2.0 nanograms (ng)/ $\mu \mathrm{L}$ prior to being used in quantitative Reverse Transcription PCR (qRT-PCR) assays targeting the Wolbachia $16 S$ rRNA gene [28]. A synthetic oligonucleotide standard (Integrated DNA Technologies) was designed to calculate $16 S$ rRNA gene copies per $\mu \mathrm{L}$ using a ten-fold serial dilution (electronic supplementary material, supplementary figure 1). $16 S r R N A$ gene real time qRT-PCR reactions were prepared using $5 \mu \mathrm{L}$ of QuantiNova SYBR ${ }^{\circledR}$ Green RT-PCR Kit (Qiagen), a final concentration of $1 \mu \mathrm{M}$ of each primer, $1 \mu \mathrm{L}$ of PCR grade water and $2 \mu \mathrm{L}$ template DNA, to a final reaction volume of $10 \mu \mathrm{L}$. Prepared reactions were run on a Roche LightCycler 96 System for 15 minutes at $95^{\circ} \mathrm{C}$, followed by 40 cycles of $95^{\circ} \mathrm{C}$ for 15 seconds and $58^{\circ} \mathrm{C}$ for 30 seconds. Amplification was followed by a dissociation curve $\left(95^{\circ} \mathrm{C}\right.$ for 10 seconds, $65^{\circ} \mathrm{C}$ for 60 seconds and $97^{\circ} \mathrm{C}$ for 1 second) to ensure the correct target sequence was being amplified. Each mosquito RNA extract was run in triplicate alongside standard curves and no template controls (NTCs) and PCR results were analysed using the LightCycler ${ }^{\circledR} 6$ software (Roche Diagnostics).

Asaia detection. Asaia PCR screening was undertaken by targeting the Asaia 165 rRNA gene using primers Asafor: 5'-GCGCGTAGGCGGTTTACAC-3' and Asarev: 5'-AGCGTCAGTAATGAGCCAGGTT-3' [33, 51]. Asaia $16 S$ rRNA gene real time qRT-PCR reactions were prepared using $5 \mu \mathrm{L}$ of QuantiNova SYBR ${ }^{\circledR}$ Green RTPCR Kit (Qiagen), a final concentration of $1 \mu \mathrm{M}$ of each primer, $1 \mu \mathrm{L}$ of PCR grade water and $2 \mu \mathrm{L}$ template DNA, to a final reaction volume of $10 \mu \mathrm{L}$. Prepared reactions were run on a Roche LightCycler ${ }^{\circledR} 96$ System for 15 minutes at $95^{\circ} \mathrm{C}$, followed by 40 cycles of $95^{\circ} \mathrm{C}$ for 15 seconds and $58^{\circ} \mathrm{C}$ for 30 seconds. Amplification was followed by a dissociation curve $\left(95^{\circ} \mathrm{C}\right.$ for 10 seconds, $65^{\circ} \mathrm{C}$ for 60 seconds and $97^{\circ} \mathrm{C}$ for 1 second) to ensure the correct target sequence was being amplified. 
Statistical analysis. Normalised qRT-PCR Wolbachia $16 S r R N A$ gene copies per $\mu \mathrm{L}$ were compared using unpaired t-tests in GraphPad Prism 7.

\section{Results}

Mosquito species and Wolbachia strain prevalence rates. In addition to confirmation of species for the morphologically indistinguishable individuals within the An. gambiae complex, initial screening using diagnostic species-specific PCRs highlighted the presence of some naturally occurring hybrids between members of the An. gambiae complex. Concomitant PCR screening demonstrated the presence of Wolbachia within individuals of the An. gambiae complex, including a number of the hybrid specimens (electronic supplementary material, table S1). The composition of these hybrids was further investigated and confirmed through repeat of the normally multiplex ribosomal IGS PCR [37] in single-plex format, separating the An. gambiae s.s. / coluzzii primer set from the An. melas primer set, achieving strong amplification for both target sequences (figure 1a) and confirmed for some representative samples through Sanger sequencing and phylogenetic analysis of both IGS PCR products from the same individuals (figure $1 b$ ). The further use of PCR amplification, Sanger sequencing and phylogenetic analysis of the ribosomal ITS2 (Figure 1c) and mitochondrial COI, COII (figure 2) and ND4-ND5 (Figure 3) genes was able to confirm both the mosquito species identity for individuals of interest and the composition of hybrids, with mitochondrial gene analysis indicating the maternal species identity. Prevalence rates of natural Wolbachia strains were variable depending on Anopheles species and location (table 1). Wolbachia strains were detected in An. gambiae s.s. mosquitoes from the Faranah region with prevalence rates ranging from $0.0-2.8 \%$. In the Maferinyah region, from individuals collected in Senguelen, Wolbachia strains were detected in An. melas (11.6\% - 16/138) and in An. gambiae s.s.melas hybrids ( $40.0 \%$ prevalence - 6/15). Interestingly, Wolbachia was not found in any of the 4 An. gambiae s.s., 18 An. coluzzii, 2 An. coluzzii-gambiae s.s. hybrids or an An. coluzzii-melas hybrid collected from Senguelen, suggesting Wolbachia strains are not currently widespread across all members of the An. gambiae complex in this location. Phylogenetic hybrid composition analysis combined with Wolbachia screening highlighted the majority of An. gambiae s.s.-melas hybrids collected from Senguelen had An. melas mothers (8/12 An. melas by mitochondrial analysis), with 4/6 Wolbachia positive hybrids having An. melas as the maternal species. These results, combined with the prevalence of maternally inherited Wolbachia in the An. melas individuals and not in the An. gambiae s.s. individuals from this location suggests the Wolbachia in this population has most likely originated from An. melas. Wolbachia-negative An. gambiae s.s.-melas hybrids were also confirmed for two specimens from Fandie (with An. gambiae s.s. mitochondrial results) and a Wolbachia-negative An. coluzziigambiae s.s. hybrid (maternally An. coluzzii) from Maferinyah.

A Wolbachia strain was also found in a single female of an unclassified Anopheles species from Senguelen. Sanger sequencing and BLAST analysis of the ITS2 region revealed this Anopheles sp. ' $X$ ' was most similar to Anopheles sp. 7 BSL-2014 (GenBank accession number KJ522819.1) but at only 93.2\% sequence identity, and An. theileri (GenBank accession number MH378771.1) with 90.9\% sequence identity (both full query coverage). Phylogenetic analysis of the ribosomal ITS2 region and mitochondrial COI and ND4-ND5 regions for An. sp. ' $\mathrm{X}$ ' (Figure $4 a, b$ and $c$ ) revealed that this species is from the Myzomyia Series, within the Cellia Subgenus of Anopheles, with agreement for this placement across all three phylogenies. The ITS2 region gave the greatest discrimination for this species, however, currently no other sequences from this species are available in order to classify it any further than to Series level and closest to, but distinct from, sequences denoted Anopheles sp. 7, another as yet undetermined Anopheles species [52]. Mosquito ribosomal and mitochondrial gene sequences were deposited in GenBank and accession numbers obtained (electronic supplementary material, table S2).

Wolbachia strain typing. Although amplification of the Wolbachia $16 S r R N A$ fragments of the natural strain in An. gambiae s.s. from the Faranah region was possible, sequences obtained were of insufficient quality for further analysis. Furthermore, no wsp gene amplification was possible from An. gambiae s.s. from the Faranah region. In contrast, Wolbachia $16 S r R N A$ (figure 5) and wsp sequences (figure 6) were generated from both $A n$. melas / An. gambiae s.s.-melas hybrids and An. sp. X collected from Senguelen in the Maferinyah region. Analysis of Wolbachia $16 S$ rRNA sequences obtained from An. melas and An. gambiae s.s.-melas hybrid individuals highlighted the occurrence of superinfections within this population, with the presence of multiple Wolbachia strains being indicated. The Wolbachia $16 S$ sequences from some An. melas and hybrid 
bioRxiv preprint doi: https://doi.org/10.1101/772855; this version posted November $17,2020$. The copyright holder for this preprint (which was not certified by peer review) is the author/funder, who has granted bioRxiv a license to display the preprint in perpetuity. It is made available under aCC-BY-NC-ND 4.0 International license.

individuals produced consensus sequences which were most closely related to Wolbachia strains of Supergroup A (such as $w \mathrm{Mel}, w \mathrm{AlbA}$ and $w \mathrm{Au}$ ) (wAnga-Guinea-A), of which two different A strains (A1 and A2) could be determined in different individuals. In contrast, other An. melas and hybrid specimens produced Wolbachia $16 S$ consensus sequences which grouped clearly with Supergroup B strains (wAnga-Guinea-B), also with two differing B strains able to be determined (B1 and B2) (figure 5). In addition, the sequence chromatograms from other An. melas and hybrid individuals consistently demonstrated mixed bases in the positions of variation between the $w$ Anga-Guinea-A and $w$ Anga-Guinea-B strains, with agreement both between forward and reverse sequence traces from the same individuals, as well as across multiple individuals, suggesting the presence of superinfections of both Supergroup A and B Wolbachia strains within these individuals.

To try to further understand the complex picture of Wolbachia strains in the An. melas and An. gambiae s.s.-melas hybrids from Senguelen, certain An. melas and hybrid individuals were selected for repeat Wolbachia $16 \mathrm{~S}$ sequencing, including representative samples where the original analysis suggested the presence of a $w$ AngaGuinea A and B strain superinfection and where there was apparent dominance of one or other strain. Comparison of the repeat $16 S$ sequences to the original analysis for each individual suggested that the presence of superinfections was genuinely evident but that it wasn't possible to confidently separate An. melas and hybrid individuals into wAnga-Guinea-A only, wAnga-Guinea-B only, or superinfected groups, with the dominance of a particular strain over another, on the basis of $16 \mathrm{~S}$ sequencing analysis alone. This was due to variation in the dominance of $w$ Anga-Guinea-A or $w$ Anga-Guinea-B sequences in chromatograms, suggesting the possibility that any difference could be due to normal technical variations in the processes of amplification and sequencing (e.g. the apparent dominance of either an A or B strain in chromatograms from a particular PCR product could be due to the chance of variation in amplification efficiency and the resultant sequencing signal strength), rather than detection of a repeatable biological difference, with consistent dominance of a particular strain variant over another within each individual. This repeat analysis on a small sub-group suggested that Wolbachia superinfections were likely in all the An. melas or hybrid individuals where this subsequent $16 S$ analysis was possible.

This, combined with the overall results from the Wolbachia $16 S$ analysis from all infected individuals suggested superinfections were widespread in Wolbachia positive individuals but there did not currently appear to be a clear dominant strain, or strain variant, which could be identified with greater relative occurrence or apparent density (through consistent stronger sequencing signal strength) to the other strain(s) present in individuals from this population. This complexity was also mirrored when looking between An. melas and the hybrid specimens, with no clear distinction in the Wolbachia strain variants apparent in each group. Unfortunately, further comparative analysis of differing strains in An. melas and hybrid individuals utilising the Wolbachia wsp gene locus was not possible, as wsp sequence could only be successfully obtained from one An. melas individual, where $16 \mathrm{~S}$ analysis had indicated the presence of $w$ Anga-Guinea-A1 only. This wsp sequence matched allele 23 within the Wolbachia MLST database (table 2), demonstrating that it is identical to the wsp sequences obtained from 20 other Supergroup A Wolbachia isolates contained within the database.

Phylogenetic analysis of both Wolbachia $16 S$ and wsp gene fragments from An. sp. X indicated that the wAnsX strain is most closely related to Wolbachia strains of Supergroup B (such as $w \mathrm{Pip}, w \mathrm{AlbB}, w \mathrm{Ans} A, w \mathrm{AnM}, w \mathrm{Ma}$ and $w \mathrm{No}$ ). Typing of the $w \mathrm{Ans} \mathrm{X} w s p$ nucleotide sequence highlighted that there were no exact matches to $w s p$ alleles currently in the Wolbachia MLST database (https://pubmlst.org/Wolbachia/), and only one of the four hypervariable regions (HVRs) matched a known sequence (HVR3: allele 3). All Wolbachia gene sequences of sufficient quality to generate a consensus were deposited into GenBank and accession numbers obtained (electronic supplementary material, supplementary tables S3 and S4).

Wolbachia MLST was undertaken to attempt to provide more accurate strain discrimination and phylogenies. This was successfully done for the novel Anopheles Wolbachia strains wAnga-Guinea-A1 and wAnsX but no amplification was seen for any of the five MLST genes from Wolbachia-infected An. gambiae s.s. from Faranah, and successful sequencing of complete MLST profiles of sufficient quality for onward analysis was not possible from any further Wolbachia positive An. melas or An. gambiae s.s.-melas hybrid individuals from Senguelen. MLST gene fragment amplification was variable for $w$ Anga-Guinea strains found in An. melas and An. gambiae s.s.-melas hybrids. Even for $w$ Anga-Guinea-A1, the use of $h c p A$ 'A strain specific' primers (hcpA_F1: GAAATARCAGTTGCTGCAAA, hcpA_AspecR1: TTCTARYTCTTCAACCAATGC) was required 
to generate sequence of sufficient quality for analysis of the hcpA gene and to therefore complete the Wolbachia MLST profile for this An. melas sample. Despite this use of Supergroup specific primers, there was still some indication of a possible mixed strain from the chromatograms generated from sequencing the A strain-specific hcp A PCR product, through mixed bases at two positions on both the forward and reverse reads, although a consensus could still be obtained through agreement of the strongest base at each position across reads. The resultant MLST allelic profile for wAnga-Guinea-A1 (table 2) was closest to the profile for Strain Type 13, with the variation occurring in the two positions of mixed bases in the hcpA locus, being closest to $h c p A$ allele 1, except for a change from $\mathrm{G}$ to $\mathrm{A}$ at position 313 and from $\mathrm{A}$ to $\mathrm{G}$ at position 319 on this locus (table 2). This may indicate the presence of both $h c p A$ allele 1 and an $h c p A$ variant. Even if $w$ Anga-Guinea-A1 were identical to Strain Type 13, of the 19 records available on the MLST database (all Supergroup A), no other isolates with this strain type, where host information had been provided, were found in mosquito species. Concatenation of the MLST loci and phylogenetic analysis also confirms wAnga-Guinea-A1 is closest to strains belonging to Supergroup A, including $w \mathrm{Mel}$ and $w \mathrm{AlbA}$ (as also suggested by $16 \mathrm{~S}$ and $w s p$ gene phylogenies). For $w \mathrm{Ans} \mathrm{X}$, new alleles for all five MLST gene loci (sequences differed from those currently present in the MLST database), and the therefore novel allelic profile, confirms the diversity of this novel Wolbachia strain (table 2). The phylogeny of $w$ Ans X based on concatenated sequences of all five MLST gene loci confirms this strain clusters within Supergroup B and further demonstrates that it is distinct from other currently available strain profiles (figure 7). Consistent with previous studies looking at novel Wolbachia strains in Anopheles species using MLST [24], these results highlight the lack of concordance between Wolbachia strain phylogeny and their insect hosts across diverse geographical regions.

Wolbachia strain densities and relative abundance. The relative densities of Wolbachia strains were estimated using qRT-PCR targeting the $16 S \mathrm{SRNA}$ gene after first standardising total RNA (ng per reaction). This allowed direct comparisons between phylogenetically diverse Anopheles species and accounts for variation in mosquito body size and RNA extraction efficiency between samples. This also allows a comparison to another novel natural Wolbachia strain present in $C x$. watti (termed wWat strain) collected in Maferinyah, contemporaneously with the Anopheles specimens. $16 S r R N A$ qRT-PCR analysis revealed a mean of $1.50 \mathrm{E}+04$ $(+/-4.37 \mathrm{E}+03) 16 S r R N A$ copies/ $\mu \mathrm{L}$ for the $w A$ ns $\mathrm{X}$ strain in the single individual (figure 8, electronic supplementary material, supplementary table S5). Lower mean densities were found for the wAnga-Guinea strains in An. melas individuals $(\mathrm{n}=14)$ and An. gambiae s.s.-melas hybrids $(\mathrm{n}=4)$ with $8.20 \mathrm{E}+02(+/-2.90 \mathrm{E}+02)$ and $1.41 \mathrm{E}+02(+/-3.95 \mathrm{E}+01) 16 S r R N A$ copies/ $\mu \mathrm{L}$ respectively. The densities were compared to the $w$ Wat strain in $C x$. watti females also collected in the Maferinyah region with a mean density of $2.37 \mathrm{E}+04(+/-5.99 \mathrm{E}+03)$. The density of the $w$ Wat strain was significantly higher than the wAnga-Guinea strains found in An. melas and hybrids (Unpaired T-test, $\mathrm{p}=0.002$ ). Individual An. gambiae s.s. extracts from the Faranah region that were identified as Wolbachia-infected by amplification of the $16 S r R N A$ gene [44] did not result in any $16 S r R N A$ qRT-PCR amplification, suggesting a very low titre Wolbachia strain present in these individuals.

Wolbachia and Asaia co-infections. Individual mosquitoes shown to be infected with the wAnsX or wAngaGuinea strains were screened for the presence of Asaia bacteria using qRT-PCR. Co-infections were detected in all An. melas ( $\mathrm{n}=14$, mean Asaia $16 S$ rRNA Ct value $=30.60+/-2.02)$, all An. gambiae s.s. - melas hybrids $(\mathrm{n}=4$, mean Asaia $16 S r R N A$ Ct value $=26.32+/-3.54)$ and in the single An. species X (Asaia $16 S r R N A$ Ct value $=34.92)$ (electronic supplementary material, supplementary table S5).

\section{Discussion}

Endosymbiotic Wolbachia bacteria are particularly widespread through insect populations but were historically considered absent from the Anopheles genera [19]. The discovery of additional novel natural strains of Wolbachia in Anopheles species suggests that the prevalence and diversity has been significantly under-reported to date. Since 2014, there have been several reports of detection of Wolbachia strains in major malaria vectors, such as sibling species in the An. gambiae complex [25, 26, 28-30] and An. moucheti [26]. This study provides evidence for Wolbachia strains in An. melas, a species within the An. gambiae complex, which can be an important local vector of malaria in West-African coastal areas where it breeds in brackish water, mangrove forests and salt marshes [53,54]. It's importance as a local malaria vector was shown in Equatorial Guinea where the average number of malaria infective An. melas bites/person/year was recorded at up to 130 [55]. The finding of natural An. gambiae s.s.-melas hybrids in this study appears highly unusual, with 
bioRxiv preprint doi: https://doi.org/10.1101/772855; this version posted November $17,2020$. The copyright holder for this preprint (which was not certified by peer review) is the author/funder, who has granted bioRxiv a license to display the preprint in perpetuity. It is made available under aCC-BY-NC-ND 4.0 International license.

published accounts of hybridisation between members of the An. gambiae complex seeming to agree that detection of hybrids in wild populations is relatively rare [56], and when it does occur, seems most often to be a combination of hybrids between An. gambiae s.s., An. coluzzii or An. arabiensis. Historical reports of An. gambiae s.s.-melas hybrids were also in West Africa but with laboratory colonies, giving variable results for ongoing success of hybrid colonies [57-59]. Interestingly colonised An. melas and F1 hybrid larvae were able to be reared in distilled water in the laboratory, rather than requiring a higher salinity content as might be expected from the natural ecology of An. melas [59]. As An. melas is more geographically restrained and has a more defined ecological niche than other members of the An. gambiae complex, natural hybrids composed of these constituent species are arguably less likely to occur, with fewer areas of sympatry. Natural hybrids may also be underestimated [60] due to sampling bias with a greater proportion of studies focussing on the more widely distributed major anthrophilic malaria vectors, An. gambiae and An. arabiensis [61].

Hybrid detection is also dependent on the methodology used for species identification and the format of species-specific diagnostic assays $[37,56]$. Our testing highlighted that amplification and clarity of hybrid detection was improved with use of the ribosomal IGS PCR primers[37] for each species in single-plex format, rather than the standard higher-throughput multiplex format, where primers for multiple members of the An. gambiae complex are included at the same time, with different product sizes for species discrimination. This is unsurprising due to the designed aims of the multiplex assay, and potential variations in reaction efficiency between species, particularly when hybridised, which were highlighted in the original publication [37]. However, this could potentially result in reduced detection of natural hybrids, compared to the apparent detection of individual species, when used for widespread screening and species identification. Sanger sequencing of the single-plex species-specific IGS PCR products for representative hybrid samples enabled confirmation of the hybridisation and the avoidance of doubt from any possibility of specificity problems [37, 56], before further confirmation was obtained through subsequent sequencing and phylogenetic analysis of other gene fragments.

Genetic divergence also likely affects interspecific hybrids and the original delineation of the member species within the An. gambiae complex was concluded on the basis of hybrid male sterility from early crossing experiments [61]. However, the full extent and impacts of interspecific hybridisation between members of the complex is still under investigation and debate [60]. An. gambiae s.s. and An. melas have a greater degree of genetic divergence from one another when compared to other members of the complex (such as An. gambiae s.s., An. coluzzii and An. arabiensis) and An. melas groups separately and more closely to An. merus and An. quadriannulatus sequences. Even within An. melas, species-specific microsatellite markers and mitochondrial genetic analysis of geographically distinct populations suggested there was species level divergence between different populations, resulting in three distinct major clusters; Bioko Island, Western mainland and Southern mainland African populations (with mainland population division occurring in Cameroon) [61]. In the context of the results of this study, our An. melas would be included in the Western mainland cluster (this is supported by our phylogenetic analysis). Following the discovery of Wolbachia in this population, it would be interesting to investigate whether Wolbachia strains were also present in other An. melas geographic clusters, and whether the CI phenotype was evident in some or all of these strains. If stable Wolbachia infections were present in some populations but not others, it also raises the question of the length of time Wolbachia may have been present in this species and whether Wolbachia infections may be having an influence on the host population genetics and affecting genetic divergence and speciation over time.

The discovery of the $w$ Ans $\mathrm{X}$ strain led to retrospective confirmation of the host mosquito species using Sanger sequencing. In this study and previous studies, thorough and accurate molecular identification is important given the difficulties of morphological identification, the potential for currently unrecognised cryptic species $[52,62]$ and potential for inaccuracies for certain species where only diagnostic species PCR-based methods are used for molecular identification [63]. Phylogenetic analysis and confident species discrimination is dependent on the sequences available for comparison at the time. Sequencing and phylogenetic analysis of all three regions for this specimen indicated placement within the Cellia Subgenus and Myzomyia Series of Anopheles, with the greatest number of closely related comparative sequences available for comparison in the ITS2 region. Our analysis revealed that this species is closest to Anopheles sp. 7, followed by An. theileri from sequences currently available. Anopheles sp. 7 BSL-2014 was collected in the Western Kenyan Highlands, with 1 of 23 specimens Plasmodium falciparum ELISA sporozoite and PCR positive[52]. An. theileri was collected in 
the Democratic Republic of Congo [64] and was found to be infected with Plasmodium sporozoites in eastern Zambia [65].

The results of this study also highlight the requirement to provide as much genetic information and confirmation as possible for a newly discovered strain of Wolbachia (particularly low-density infections). The first discovery of Wolbachia strains in wild An. gambiae populations in Burkina Faso resulted from sequencing of the $16 S r R N A$ gene rather than screening using Wolbachia-specific genes [25]. A more recent comprehensive analysis through screening of An. gambiae genomes (Ag1000G project) concluded that determining whether a Wolbachia strain is present in a given host based on the sequencing of one gene fragment (often $16 S r R N A$ ) is problematic and caution should be taken [31]. In this study, we were only able to amplify a Wolbachia $16 S$ $r R N A$ gene fragment from An. gambiae s.s., which is consistent with numerous recent studies in which low density strains have been detected $[27,30]$. As a result, caution must be taken in drawing conclusions on the stability of infection and biological significance. Other explanations for the amplification of $16 S r R N A$ gene fragments include Wolbachia DNA insertions into an insect chromosome or contamination from non-mosquito material such as ectoparasites or plants [31]. In contrast to previous studies, we extracted RNA increasing the chances that detection of the $16 S r R N A$ gene is from actively expressed Wolbachia and indicating amplification is more likely of bacterial gene origin (rather than through integration into the host genome). However, these results are consistent with previous studies in which every Wolbachia $16 S$ rRNA amplicon and sequence attributed to An. gambiae s.s. is unique and appears at very low density [31].

The densities of the wAnga-Guinea and wAnsX strains detected in Senguelen (measured using qRT-PCR) are significantly higher than Wolbachia detected in An. gambiae s.s. from Faranah (which were not detectable using this qRT-PCR assay targeting the $16 S r R N A$ gene). The $w$ Anga-Guinea strains appear to have both an intermediate prevalence rate and density and further studies are required to elucidate the relative density contribution and possible differential localisation of these Wolbachia strains within the mosquito host, whether these strains may be influencing host population genetics (including the occurrence of natural hybrids and the intraspecific diversity within An. melas) and investigate these strains across more diverse geographical areas. Caution and further investigation is also required for the $w A n s X$ strain as this was detected from the only collected individual of this unclassified Anopheles species. The detection of Wolbachia-Asaia co-infections in all individuals was in contrast to our previous study [26] but Asaia can be environmentally acquired at different mosquito life stages and the prevalence and density was significantly variable across different Anopheles species and locations [26]. These contrasting results suggest a complex association between these two bacterial species in wild Anopheles mosquito populations and given that Asaia is environmentally acquired, this association will be highly location dependent.

Wolbachia strains in An. species A (wAnsA) and An. moucheti (wAnM) [26], and now An. melas (wAnga-GuineaA1) and $A n . s p . X(w A n s X)$, have complete MLST and $w s p$ profiles and are at significantly higher densities when compared to strains detected in An. gambiae s.s. from the same countries. As Wolbachia density is strongly correlated with arbovirus inhibition in Aedes mosquitoes [5, 7, 11, 12], higher density strains in Anopheles species would be predicted to have a greater impact on malaria transmission in field populations. In this study, we screened for $P$. falciparum infection and found very low prevalence rates ( $<1 \%$; data not shown) preventing any statistical analysis on Wolbachia-Plasmodium interactions. This study and previous studies measuring a direct impact on Plasmodium infection in wild populations are dependent on parasite infection rates which can be low even in malaria-endemic areas [26] and particularly for the infective sporozoite stage [66]. Low pathogen prevalence rates are also limiting factors in assessing the effect of natural strains of Wolbachia on arboviruses in wild mosquito populations [67]. In addition to looking at effects on Plasmodium prevalence in field populations, further work should look to undertake vector competence experiments with colonised populations and to determine if these Wolbachia strains are present in tissues such as the midgut and salivary glands which are critical to sporogony. Further studies are also needed to determine if the wAngaGuinea strains are maternally transmitted given our results would suggest they are likely to be from the $A n$. melas, rather than from An. gambiae s.s. Furthermore, an assessment of how these Wolbachia strains are being maintained in field populations is needed, and to determine if the CI reproductive phenotype can be induced by these strains (and if it affects viability of subsequent generations). As the chances of success of Wolbachia transinfection experiments can be improved by the adaptation of the Wolbachia strain to the target host genetic background[68], this may imply favourable potential for wAnga-Guinea transinfection experiments and successful establishment of a stable Wolbachia infection within An. gambiae s.s. colonies, in addition to An. 
bioRxiv preprint doi: https://doi.org/10.1101/772855; this version posted November $17,2020$. The copyright holder for this preprint (which was not certified by peer review) is the author/funder, who has granted bioRxiv a license to display the preprint in perpetuity. It is made available under aCC-BY-NC-ND 4.0 International license.

melas. If achievable, this would be a big step forward in determining whether these strains (which appear relatively higher density than Wolbachia previously detected in the An. gambiae complex) could reduce malaria transmission through Wolbachia-based biocontrol strategies.

\section{Conclusion}

Although the debate continues over the biological significance (or even presence of natural strains in the An. gambiae complex), this study provides strong evidence of additional novel strains with relatively higher density infections, in addition to Wolbachia positive natural hybrids in the An. gambiae complex, and may reflect the under-reporting of natural strains in the Anopheles genus. The presence of Wolbachia superinfections increases the complexity of phylogenetic characterisation of individual strains and the determination of the relative contribution of each strain to the overall density. There are previous studies showing natural Wolbachia superinfections in wild mosquito populations such as Ae. albopictus [69] and superinfections have been generated in mosquitoes used for biocontrol strategies $[7,70]$ indicating superinfections can form stable associations with mosquito hosts. Candidate Wolbachia strains for mosquito biocontrol strategies require synergistic phenotypic effects to impact the transmission of mosquito-borne pathogens and further studies are needed to determine if these strains would induce CI and what effects they may have on host fitness. Whether these Wolbachia superinfections can inhibit Plasmodium parasites [28, 29] or influence the ability to transinfect other Wolbachia strains for population suppression and replacement strategies [71] remains to be determined but further investigation is warranted.

Acknowledgments. The following people are thanked for their efforts in the original collection of mosquitoes: Moussa Sylla, Gnepou Camara, Louisa Messenger, Patrick Heard, Yaya Barry, Denka Camara, and Ismael Yansane. This publication made use of the PubMLST website (https://pubmlst.org/Wolbachia/) sited at the University of Oxford (Iolley \& Maiden 2010, BMC Bioinformatics, 11:595). The development and maintenance of this site has been funded by the Wellcome Trust.

Ethical Statement. Mosquito collection protocols were reviewed and approved by the Comite National d'Ethique pour la Recherche en Sante (030/CNERS/17) and the institutional review boards (IRB) of the London School of Hygiene and Tropical Medicine (\#14798 and \#15127) and the Centers for Disease Control and Prevention, USA (2018-086); all study procedures were performed in accordance with relevant guidelines and regulations. Fieldworkers participating in human landing catches were provided with malaria prophylaxis for the duration of the study.

Funding Statement. CLJ and TW were supported by Wellcome Trust/Royal Society grants awarded to TW (101285/Z/13/Z): http://www.wellcome.ac.uk; https://royalsociety.org. SRI was supported by the President's Malaria Initiative (PMI)/CDC.

Data Accessibility. The datasets supporting this article have been uploaded as part of the Supplementary Material and all the sequencing data generated is available in GenBank with accession numbers as shown in the relevant supplementary tables.

Competing interests. We have no competing interests.

Authors' Contributions. C.L.J. designed the study, carried out the analyses and drafted the manuscript. C.C-U. performed field work, carried out preliminary laboratory analysis and reviewed the manuscript. A.H.B. supervised field work and reviewed the manuscript. C.S. performed field work and reviewed the manuscript. E.K.L. supervised fieldwork and reviewed the manuscript. M.K. performed field work and reviewed the manuscript. S.R.L. supervised fieldwork and reviewed the manuscript. T.W. designed the study, carried out analyses, drafted the manuscript and provided overall supervision.

References

1 Zug, R., Hammerstein, P. 2012 Still a host of hosts for Wolbachia: analysis of recent data suggests that $40 \%$ of terrestrial arthropod species are infected. PLoS One. 7, e38544. (10.1371/journal.pone.0038544)

2 Glaser, R. L., Meola, M. A. 2010 The native Wolbachia endosymbionts of Drosophila melanogaster and Culex quinquefasciatus increase host resistance to West Nile virus infection. PLoS One. 5, e11977. (10.1371/journal.pone.0011977) 3 Mousson, L., Zouache, K., Arias-Goeta, C., Raquin, V., Mavingui, P., Failloux, A. B. 2012 The native Wolbachia symbionts limit transmission of dengue virus in Aedes albopictus. PLoS Negl Trop Dis. 6, e1989. (10.1371/journal.pntd.0001989) 
4 Silva, J. B. L., Magalhaes Alves, D., Bottino-Rojas, V., Pereira, T. N., Sorgine, M. H. F., Caragata, E. P., Moreira, L. A. 2017 Wolbachia and dengue virus infection in the mosquito Aedes fluviatilis (Diptera: Culicidae). PLoS One. 12, e0181678. (10.1371/journal.pone.0181678)

5 Walker, T., Johnson, P. H., Moreira, L. A., Iturbe-Ormaetxe, I., Frentiu, F. D., McMeniman, C. J., Leong, Y. S., Dong, Y., Axford, J., Kriesner, P., et al. 2011 The wMel Wolbachia strain blocks dengue and invades caged Aedes aegypti populations. Nature. 476, 450-U101. (10.1038/nature10355)

6 Iturbe-Ormaetxe, I., Walker, T., Neill, S. L. O. 2011 Wolbachia and the biological control of mosquito-borne disease. Embo Reports. 12, 508-518. (10.1038/embor.2011.84)

7 Joubert, D. A., Walker, T., Carrington, L. B., De Bruyne, J. T., Kien, D. H., Hoang Nle, T., Chau, N. V., Iturbe-Ormaetxe, I., Simmons, C. P., O'Neill, S. L. 2016 Establishment of a Wolbachia Superinfection in Aedes aegypti Mosquitoes as a Potential Approach for Future Resistance Management. PLoS Pathog. 12, e1005434. (10.1371/journal.ppat.1005434)

8 Moreira, L. A., Iturbe-Ormaetxe, I., Jeffery, J. A., Lu, G., Pyke, A. T., Hedges, L. M., Rocha, B. C., Hall-Mendelin, S., Day, A., Riegler, M., et al. 2009 A Wolbachia symbiont in Aedes aegypti limits infection with dengue, Chikungunya, and Plasmodium. Cell. 139, 1268-1278. (10.1016/j.cell.2009.11.042)

9 Bian, G., Xu, Y., Lu, P., Xie, Y., Xi, Z. 2010 The endosymbiotic bacterium Wolbachia induces resistance to dengue virus in Aedes aegypti. PLoS Pathog. 6, e1000833. (10.1371/journal.ppat.1000833)

10 Blagrove, M. S., Arias-Goeta, C., Failloux, A. B., Sinkins, S. P. 2012 Wolbachia strain wMel induces cytoplasmic incompatibility and blocks dengue transmission in Aedes albopictus. Proc Natl Acad Sci U S A. 109, 255-260.

(10.1073/pnas.1112021108)

11 Ant, T. H., Herd, C. S., Geoghegan, V., Hoffmann, A. A., Sinkins, S. P. 2018 The Wolbachia strain wAu provides highly efficient virus transmission blocking in Aedes aegypti. PLOS Pathog. 14, e1006815. (10.1371/journal.ppat.1006815) 12 Fraser, J. E., De Bruyne, J. T., Iturbe-Ormaetxe, I., Stepnell, J., Burns, R. L., Flores, H. A., O'Neill, S. L. 2017 Novel Wolbachia-transinfected Aedes aegypti mosquitoes possess diverse fitness and vector competence phenotypes. PLoS Pathog. 13, e1006751. (10.1371/journal.ppat.1006751)

13 McMeniman, C. J., Lane, R. V., Cass, B. N., Fong, A. W., Sidhu, M., Wang, Y. F., O'Neill, S. L. 2009 Stable introduction of a life-shortening Wolbachia infection into the mosquito Aedes aegypti. Science. 323, 141-144. (10.1126/science.1165326) 14 Hoffmann, A. A., Montgomery, B. L., Popovici, J., Iturbe-Ormaetxe, I., Johnson, P. H., Muzzi, F., Greenfield, M., Durkan, M., Leong, Y. S., Dong, Y., et al. 2011 Successful establishment of Wolbachia in Aedes populations to suppress dengue transmission. Nature. 476, 454-457. (10.1038/nature10356)

15 Frentiu, F. D., Zakir, T., Walker, T., Popovici, J., Pyke, A. T., van den Hurk, A., McGraw, E. A., O'Neill, S. L. 2014 Limited dengue virus replication in field-collected Aedes aegypti mosquitoes infected with Wolbachia. PLoS Negl Trop Dis. 8, e2688. (10.1371/journal.pntd.0002688)

16 O'Neill, S. L., Ryan, P. A., Turley, A. P., Wilson, G., Retzki, K., Iturbe-Ormaetxe, I., Dong, Y., Kenny, N., Paton, C. J., Ritchie, S. A., et al. 2018 Scaled deployment of Wolbachia to protect the community from dengue and other Aedes transmitted arboviruses. Gates Open Res. 2, 36. (10.12688/gatesopenres.12844.3)

17 Ryan, P. A., Turley, A. P., Wilson, G., Hurst, T. P., Retzki, K., Brown-Kenyon, J., Hodgson, L., Kenny, N., Cook, H., Montgomery, B. L., et al. 2019 Establishment of wMel Wolbachia in Aedes aegypti mosquitoes and reduction of local dengue transmission in Cairns and surrounding locations in northern Queensland, Australia. Gates Open Res. 3, 1547. (10.12688/gatesopenres.13061.1)

18 Nazni, W. A., Hoffmann, A. A., Noor Afizah, A., Cheong, Y. L., Mancini, M. V., Golding, N., Kamarul, G. M. R., Arif, M. A. K., Thohir, H., NurSyamimi, H., et al. 2019 Establishment of Wolbachia Strain w AlbB in Malaysian Populations of Aedes aegypti for Dengue Control. Curr Biol. 29, 4241-4248 e4245. (10.1016/j.cub.2019.11.007)

19 Walker, T., Moreira, L. A. 2011 Can Wolbachia be used to control malaria? Memorias Do Instituto Oswaldo Cruz. 106, $212-$ 217.

20 Hughes, G. L., Koga, R., Xue, P., Fukatsu, T., Rasgon, J. L. 2011 Wolbachia infections are virulent and inhibit the human malaria parasite Plasmodium falciparum in Anopheles gambiae. PLoS Pathog. 7, e1002043. (10.1371/journal.ppat.1002043) 21 Hughes, G. L., Vega-Rodriguez, J., Xue, P., Rasgon, J. L. 2012 Wolbachia strain wAlbB enhances infection by the rodent malaria parasite Plasmodium berghei in Anopheles gambiae mosquitoes. Appl Environ Microbiol. 78, 1491-1495.

(10.1128/AEM.06751-11)

22 Murdock, C. C., Blanford, S., Hughes, G. L., Rasgon, J. L., Thomas, M. B. 2014 Temperature alters Plasmodium blocking by Wolbachia. Sci Rep. 4, 3932. (10.1038/srep03932)

23 Hughes, G. L., Rivero, A., Rasgon, J. L. 2014 Wolbachia can enhance Plasmodium infection in mosquitoes: implications for malaria control? PLoS Pathog. 10, e1004182. (10.1371/journal.ppat.1004182)

24 Bian, G., Joshi, D., Dong, Y., Lu, P., Zhou, G., Pan, X., Xu, Y., Dimopoulos, G., Xi, Z. 2013 Wolbachia invades Anopheles stephensi populations and induces refractoriness to Plasmodium infection. Science. 340, 748-751. (10.1126/science.1236192) 25 Baldini, F., Segata, N., Pompon, J., Marcenac, P., Shaw, W. R., Dabire, R. K., Diabate, A., Levashina, E. A., Catteruccia, F. 2014 Evidence of natural Wolbachia infections in field populations of Anopheles gambiae. Nat Commun. 5, 3985.

(10.1038/ncomms4985)

26 Jeffries, C. L., Lawrence, G. G., Golovko, G., Kristan, M., Orsborne, J., Spence, K., Hurn, E., Bandibabone, J., Tantely, L. M., Raharimalala, F. N., et al. 2018 Novel Wolbachia strains in Anopheles malaria vectors from Sub-Saharan Africa.

Wellcome Open Res. 3, 113. (10.12688/wellcomeopenres.14765.2) 
bioRxiv preprint doi: https://doi.org/10.1101/772855; this version posted November 17, 2020. The copyright holder for this preprint (which was not certified by peer review) is the author/funder, who has granted bioRxiv a license to display the preprint in perpetuity. It is made available under aCC-BY-NC-ND 4.0 International license.

27 Niang, E. H. A., Bassene, H., Makoundou, P., Fenollar, F., Weill, M., Mediannikov, O. 2018 First report of natural Wolbachia infection in wild Anopheles funestus population in Senegal. Malar J. 17, 408. (10.1186/s12936-018-2559-z) 28 Gomes, F. M., Hixson, B. L., Tyner, M. D. W., Ramirez, J. L., Canepa, G. E., Alves, E. S. T. L., Molina-Cruz, A., Keita, M., Kane, F., Traore, B., et al. 2017 Effect of naturally occurring Wolbachia in Anopheles gambiae s.l. mosquitoes from Mali on Plasmodium falciparum malaria transmission. Proc Natl Acad Sci U S A. 114, 12566-12571. (10.1073/pnas.1716181114) 29 Shaw, W. R., Marcenac, P., Childs, L. M., Buckee, C. O., Baldini, F., Sawadogo, S. P., Dabire, R. K., Diabate, A., Catteruccia, F. 2016 Wolbachia infections in natural Anopheles populations affect egg laying and negatively correlate with Plasmodium development. Nat Commun. 7, 11772. (10.1038/ncomms11772)

30 Baldini, F., Rouge, J., Kreppel, K., Mkandawile, G., Mapua, S. A., Sikulu-Lord, M., Ferguson, H. M., Govella, N., Okumu, F. O. 2018 First report of natural Wolbachia infection in the malaria mosquito Anopheles arabiensis in Tanzania. Parasit Vectors. 11, 635. (10.1186/s13071-018-3249-y)

31 Chrostek, E., Gerth, M. 2019 Is Anopheles gambiae a Natural Host of Wolbachia? mBio. 10, (10.1128/mBio.00784-19) 32 Hughes, G. L., Dodson, B. L., Johnson, R. M., Murdock, C. C., Tsujimoto, H., Suzuki, Y., Patt, A. A., Cui, L., Nossa, C. W., Barry, R. M., et al. 2014 Native microbiome impedes vertical transmission of Wolbachia in Anopheles mosquitoes. Proc Natl Acad Sci U S A. 111, 12498-12503. (10.1073/pnas.1408888111)

33 Favia, G., Ricci, I., Damiani, C., Raddadi, N., Crotti, E., Marzorati, M., Rizzi, A., Urso, R., Brusetti, L., Borin, S., et al. 2007 Bacteria of the genus Asaia stably associate with Anopheles stephensi, an Asian malarial mosquito vector. Proc Natl Acad Sci U S A. 104, 9047-9051. (10.1073/pnas.0610451104)

34 Rossi, P., Ricci, I., Cappelli, A., Damiani, C., Ulissi, U., Mancini, M. V., Valzano, M., Capone, A., Epis, S., Crotti, E., et al. 2015 Mutual exclusion of Asaia and Wolbachia in the reproductive organs of mosquito vectors. Parasit Vectors. 8, 278. (10.1186/s13071-015-0888-0)

35 Stica, C., Jeffries, C. L., Irish, S. R., Barry, Y., Camara, D., Yansane, I., Kristan, M., Walker, T., Messenger, L. A. 2019 Characterizing the molecular and metabolic mechanisms of insecticide resistance in Anopheles gambiae in Faranah, Guinea. Malar J. 18, 244. (10.1186/s12936-019-2875-y)

36 Cansado-Utrilla, C., Jeffries, C. L., Kristan, M., Brugman, V. A., Heard, P., Camara, G., Sylla, M., Beavogui, A. H., Messenger, L. A., Irish, S. R., et al. 2020 An assessment of adult mosquito collection techniques for studying species abundance and diversity in Maferinyah, Guinea. Parasit Vectors. 13, 150. (10.1186/s13071-020-04023-3)

37 Scott, J. A., Brogdon, W. G., Collins, F. H. 1993 Identification of single specimens of the Anopheles gambiae complex by the polymerase chain reaction. Am J Trop Med Hyg. 49, 520-529. (10.4269/ajtmh.1993.49.520)

38 Santolamazza, F., Mancini, E., Simard, F., Qi, Y., Tu, Z., della Torre, A. 2008 Insertion polymorphisms of SINE200 retrotransposons within speciation islands of Anopheles gambiae molecular forms. Malar J. 7, 163. (10.1186/1475-2875-7163)

39 Beebe, N. W., Saul, A. 1995 Discrimination of all members of the Anopheles punctulatus complex by polymerase chain reaction--restriction fragment length polymorphism analysis. Am J Trop Med Hyg. 53, 478-481. (10.4269/ajtmh.1995.53.478) 40 Folmer, O., Black, M., Hoeh, W., Lutz, R., Vrijenhoek, R. 1994 DNA primers for amplification of mitochondrial cytochrome c oxidase subunit I from diverse metazoan invertebrates. Mol Mar Biol Biotechnol. 3, $294-299$.

41 Ndo, C., Antonio-Nkondjio, C., Cohuet, A., Ayala, D., Kengne, P., Morlais, I., Awono-Ambene, P. H., Couret, D., Ngassam, P., Fontenille, D., et al. 2010 Population genetic structure of the malaria vector Anopheles nili in sub-Saharan Africa. Malar J. 9, 161. (10.1186/1475-2875-9-161)

42 Deitz, K. C., Athrey, G. A., Jawara, M., Overgaard, H. J., Matias, A., Slotman, M. A. 2016 Genome-Wide Divergence in the West-African Malaria Vector Anopheles melas. G3 (Bethesda). 6, 2867-2879. (10.1534/g3.116.031906)

43 Kumar, N. P., Rajavel, A. R., Natarajan, R., Jambulingam, P. 2007 DNA barcodes can distinguish species of Indian mosquitoes (Diptera: Culicidae). J Med Entomol. 44, 1-7. (10.1603/0022-2585(2007)44[1:dbcdso]2.0.co;2)

44 Werren, J. H., Windsor, D. M. 2000 Wolbachia infection frequencies in insects: evidence of a global equilibrium? Proc Biol Sci. 267, 1277-1285. (10.1098/rspb.2000.1139)

45 Zhou, W., Rousset, F., O'Neil, S. 1998 Phylogeny and PCR-based classification of Wolbachia strains using wsp gene sequences. Proc Biol Sci. 265, 509-515. (10.1098/rspb.1998.0324)

46 LePage, D. P., Metcalf, J. A., Bordenstein, S. R., On, J., Perlmutter, J. I., Shropshire, J. D., Layton, E. M., FunkhouserJones, L. J., Beckmann, J. F., Bordenstein, S. R. 2017 Prophage WO genes recapitulate and enhance Wolbachia-induced cytoplasmic incompatibility. Nature. 543, 243-247. (10.1038/nature21391)

47 Jolley, K. A., Chan, M. S., Maiden, M. C. 2004 mlstdbNet - distributed multi-locus sequence typing (MLST) databases. BMC Bioinformatics. 5, 86. (10.1186/1471-2105-5-86)

48 Baldo, L., Dunning Hotopp, J. C., Jolley, K. A., Bordenstein, S. R., Biber, S. A., Choudhury, R. R., Hayashi, C., Maiden, M. C., Tettelin, H., Werren, J. H. 2006 Multilocus sequence typing system for the endosymbiont Wolbachia pipientis. Appl Environ Microbiol. 72, 7098-7110. (10.1128/AEM.00731-06)

49 Kumar, S., Stecher, G., Li, M., Knyaz, C., Tamura, K. 2018 MEGA X: Molecular Evolutionary Genetics Analysis across Computing Platforms. Mol Biol Evol. 35, 1547-1549. (10.1093/molbev/msy096)

50 Tamura, K., Nei, M. 1993 Estimation of the number of nucleotide substitutions in the control region of mitochond rial DNA in humans and chimpanzees. Mol Biol Evol. 10, 512-526. (10.1093/oxfordjournals.molbev.a040023)

51 Yamada, Y., Katsura, K., Kawasaki, H., Widyastuti, Y., Saono, S., Seki, T., Uchimura, T., Komagata, K. 2000 Asaia bogorensis gen. nov., sp. nov., an unusual acetic acid bacterium in the alpha-Proteobacteria. Int J Syst Evol Microbiol. 50 Pt 2, 823-829. (10.1099/00207713-50-2-823) 
52 St Laurent, B., Cooke, M., Krishnankutty, S. M., Asih, P., Mueller, J. D., Kahindi, S., Ayoma, E., Oriango, R. M., Thumloup, J., Drakeley, C., et al. 2016 Molecular Characterization Reveals Diverse and Unknown Malaria Vectors in the Western Kenyan Highlands. Am J Trop Med Hyg. 94, 327-335. (10.4269/ajtmh.15-0562)

53 Bryan, J. H., Petrarca, V., Di Deco, M. A., Coluzzi, M. 1987 Adult behaviour of members of the Anopheles gambiae complex in the Gambia with special reference to An. melas and its chromosomal variants. Parassitologia. 29, 221-249.

54 Caputo, B., Nwakanma, D., Jawara, M., Adiamoh, M., Dia, I., Konate, L., Petrarca, V., Conway, D. J., della Torre, A. 2008 Anopheles gambiae complex along The Gambia river, with particular reference to the molecular forms of An. gambiae s.s.

Malar J. 7, 182. (10.1186/1475-2875-7-182)

55 Overgaard, H. J., Reddy, V. P., Abaga, S., Matias, A., Reddy, M. R., Kulkarni, V., Schwabe, C., Segura, L., Kleinschmidt, I., Slotman, M. A. 2012 Malaria transmission after five years of vector control on Bioko Island, Equatorial Guinea. Parasit Vectors. 5, 253. (10.1186/1756-3305-5-253)

56 Temu, E. A., Hunt, R. H., Coetzee, M., Minjas, J. N., Shiff, C. J. 1997 Detection of hybrids in natural populations of the Anopheles gambiae complex by the rDNA-based, PCR method. Ann Trop Med Parasitol. 91, 963-965.

(10.1080/00034989760383)

57 Muirhead-Thomson, R. C. 1948 D.D.T. and gammexane as residual insecticides against Anopheles gambiae in African houses. Nature. 162, 109. (10.1038/163109a0)

58 Bruce-Chwatt, L. J. 1950 Recent studies on insect vectors of yellow fever and malaria in British West Africa. J Trop Med Hyg. 53, 71-79.

59 Burgess, R. W. 1962 Preliminary experiments on the hybridization of Anopheles gambiae Giles and Anopheles melas Theobald. Am J Trop Med Hyg. 11, 702-704. (10.4269/ajtmh.1962.11.702)

60 Vicente, J. L., Clarkson, C. S., Caputo, B., Gomes, B., Pombi, M., Sousa, C. A., Antao, T., Dinis, J., Botta, G., Mancini, E., et al. 2017 Massive introgression drives species radiation at the range limit of Anopheles gambiae. Sci Rep. 7, 46451.

(10.1038/srep46451)

61 Deitz, K. C., Athrey, G., Reddy, M. R., Overgaard, H. J., Matias, A., Jawara, M., Della Torre, A., Petrarca, V., Pinto, J., Kiszewski, A. E., et al. 2012 Genetic isolation within the malaria mosquito Anopheles melas. Mol Ecol. 21, 4498-4513. (10.1111/j.1365-294X.2012.05724.x)

62 Collins, F. H., Paskewitz, S. M. 1996 A review of the use of ribosomal DNA (rDNA) to differentiate among cryptic Anopheles species. Insect Mol Biol. 5, 1-9. (10.1111/j.1365-2583.1996.tb00034.x)

63 Erlank, E., Koekemoer, L. L., Coetzee, M. 2018 The importance of morphological identification of African anopheline mosquitoes (Diptera: Culicidae) for malaria control programmes. Malar J. 17, 43. (10.1186/s12936-018-2189-5)

64 Bandibabone, J., Muhigwa, J. B., Agramonte, N. M., Zawadi, B., Ombeni, L., Corredor-Medina, C., Lawrence, G. G., Chimanuka, B., Irish, S. R. 2018 Identification of Anopheles species in Sud Kivu, Democratic Republic of Congo, using molecular tools. Trans $R$ Soc Trop Med Hyg. 112, 405-407. (10.1093/trstmh/try069)

65 Lobo, N. F., St Laurent, B., Sikaala, C. H., Hamainza, B., Chanda, J., Chinula, D., Krishnankutty, S. M., Mueller, J. D., Deason, N. A., Hoang, Q. T., et al. 2015 Unexpected diversity of Anopheles species in Eastern Zambia: implications for evaluating vector behavior and interventions using molecular tools. Sci Rep. 5, 17952. (10.1038/srep17952)

66 Pombi, M., Calzetta, M., Guelbeogo, W. M., Manica, M., Perugini, E., Pichler, V., Mancini, E., Sagnon, N., Ranson, H., Della Torre, A. 2018 Unexpectedly high Plasmodium sporozoite rate associated with low human blood index in Anopheles coluzzii from a LLIN-protected village in Burkina Faso. Sci Rep. 8, 12806. (10.1038/s41598-018-31117-x)

67 Jeffries, C. L., Tantely, L. M., Raharimalala, F. N., Hurn, E., Boyer, S., Walker, T. 2018 Diverse novel resident Wolbachia strains in Culicine mosquitoes from Madagascar. Sci Rep. 8, 17456. (10.1038/s41598-018-35658-z)

68 McMeniman, C. J., Lane, A. M., Fong, A. W., Voronin, D. A., Iturbe-Ormaetxe, I., Yamada, R., McGraw, E. A., O'Neill, S. L. 2008 Host adaptation of a Wolbachia strain after long-term serial passage in mosquito cell lines. Appl Environ Microbiol. 74, 6963-6969. (10.1128/AEM.01038-08)

69 Sinkins, S. P., Braig, H. R., O'Neill, S. L. 1995 Wolbachia pipientis: bacterial density and unidirectional cytoplasmic incompatibility between infected populations of Aedes albopictus. Exp Parasitol. 81, 284-291. (10.1006/expr.1995.1119) 70 Ant, T. H., Sinkins, S. P. 2018 A Wolbachia triple-strain infection generates self-incompatibility in Aedes albopictus and transmission instability in Aedes aegypti. Parasit Vectors. 11, 295. (10.1186/s13071-018-2870-0)

71 Jeffries, C. L., Walker, T. 2016 Wolbachia Biocontrol Strategies for Arboviral Diseases and the Potential Influence of Resident Wolbachia Strains in Mosquitoes. Curr Trop Med Rep. 3, 20-25. (10.1007/s40475-016-0066-2) 
bioRxiv preprint doi: https://doi.org/10.1101/772855; this version posted November 17, 2020. The copyright holder for this preprint (which was not certified by peer review) is the author/funder, who has granted bioRxiv a license to display the preprint in perpetuity. It is made available

\section{Tables} under aCC-BY-NC-ND 4.0 International license.

Table 1

\begin{tabular}{|c|c|c|c|c|c|}
\hline Region & Location & Species & $\begin{array}{l}\text { Wolbachiat } \\
\text { individuals }\end{array}$ & Total individuals & Prevalence $(\%)$ \\
\hline Faranah & Balayani & An. gambiae s.s. & 4 & 143 & 2.80 \\
\hline Faranah & Balayani & An. coluzzii & 0 & 1 & 0.00 \\
\hline Faranah & Balayani & An. coluzzii-gambiae s.s. hybrid & 0 & 1 & 0.00 \\
\hline Faranah & Balayani & Species unknown & 0 & 1 & 0.00 \\
\hline Faranah & Faranah & An. gambiae s.s. & 0 & 26 & 0.00 \\
\hline Faranah & Faranah & An. coluzzii & 0 & 1 & 0.00 \\
\hline Faranah & Foulaya & An. gambiae s.s. & 0 & 63 & 0.00 \\
\hline Faranah & Foulaya & An. coluzzii-gambiae s.s. hybrid & 0 & 1 & 0.00 \\
\hline Faranah & Tindo & An. gambiae s.s. & 1 & 48 & 2.08 \\
\hline Faranah & Tindo & An. coluzzii & 0 & 1 & 0.00 \\
\hline Faranah & Tindo & An. coluzzii-gambiae s.s. hybrid & 0 & 2 & 0.00 \\
\hline Maferinyah & Fandie & An. coluzzii & 0 & 20 & 0.00 \\
\hline Maferinyah & Fandie & An. gambiae s.s. & 0 & 1 & 0.00 \\
\hline Maferinyah & Fandie & An. melas & 0 & 2 & 0.00 \\
\hline Maferinyah & Fandie & An. gambiae s.s.-melas hybrid & 0 & 4 & 0.00 \\
\hline Maferinyah & Fandie & An. coustani & 0 & 1 & 0.00 \\
\hline Maferinyah & Maferinyah & An. coluzzii & 0 & 6 & 0.00 \\
\hline Maferinyah & Maferinyah & An. coustani & 0 & 3 & 0.00 \\
\hline Maferinyah & Maferinyah & An. gambiae s.s. & 0 & 1 & 0.00 \\
\hline Maferinyah & Maferinyah & An. coluzzii-gambiae s.s. hybrid & 0 & 1 & 0.00 \\
\hline Maferinyah & Maferinyah & An. squamosus & 0 & 8 & 0.00 \\
\hline Maferinyah & Senguelen & An. coluzzii & 0 & 18 & 0.00 \\
\hline Maferinyah & Senguelen & An. coluzzii-melas hybrid & 0 & 1 & 0.00 \\
\hline Maferinyah & Senguelen & An. coluzzii-gambiae s.s. hybrid & 0 & 2 & 0.00 \\
\hline Maferinyah & Senguelen & An. gambiae s.s.-melas hybrid & 6 & 15 & 40.00 \\
\hline Maferinyah & Senguelen & An. coustani & 0 & 1 & 0.00 \\
\hline Maferinyah & Senguelen & An. gambiae s.s. & 0 & 4 & 0.00 \\
\hline Maferinyah & Senguelen & An. melas & 16 & 138 & 11.59 \\
\hline Maferinyah & Senguelen & An. species $X$ & 1 & 1 & 100.00 \\
\hline Maferinyah & Senguelen & An. squamosus & 0 & 1 & 0.00 \\
\hline
\end{tabular}

Table 2

\begin{tabular}{|c|c|c|c|c|c|c|c|c|c|c|c|}
\hline \multirow{2}{*}{$\begin{array}{l}\text { Mosquito } \\
\text { species }\end{array}$} & \multirow{2}{*}{$\begin{array}{l}\text { Wolbachia } \\
\text { strain }\end{array}$} & \multicolumn{5}{|c|}{$W S P$ typing allele numbers } & \multicolumn{5}{|c|}{ MLST gene allele numbers } \\
\hline & & $w s p$ & HVR1 & HVR2 & HVR3 & HVR4 & gatB & $\operatorname{cox} A$ & $h c p A$ & $f t s Z$ & $f b p A$ \\
\hline An. melas & $\begin{array}{c}\text { wAnga- } \\
\text { Guinea-A1 }\end{array}$ & 23 & 1 & 12 & 21 & 19 & 1 & 1 & $\begin{array}{c}\text { CM1 } \\
(2)^{*}\end{array}$ & 3 & 1 \\
\hline An. sp. X & wAns $X$ & 737 & 264 & 297 & 3 & 323 & 285 & 282 & 310 & 246 & 454 \\
\hline
\end{tabular}




\section{Figure and table captions}

Figure 1. An. gambiae complex PCR and phylogenetic analysis of the ribosomal IGS and ITS2 gene fragments. a) Gel electrophoresis analysis of IGS An. gambiae / melas primer split down PCR products from two representative Wolbachia positive An. gambiae s.s.-melas hybrids. b) Maximum Likelihood molecular phylogenetic analysis of sequences from IGS gambiae / melas primer split down PCR products for representative Wolbachia positive $(\mathrm{W}+)$ hybrid samples. Sequences from IGS An. melas specific primer set PCR products (blue) are shown alongside IGS An. gambiae primer set PCR products (red). The tree with the highest log likelihood (-1966.77) is shown. The tree is drawn to scale, with branch lengths measured in the number of substitutions per site. The analysis involved 22 nucleotide sequences. There was a total of 901 positions in the final dataset. Sequences obtained from GenBank for comparison are shown with their accession numbers. c) Maximum Likelihood molecular phylogenetic analysis of An. gambiae complex ITS2 sequences to demonstrate ribosomal ITS2 phylogeny of Wolbachia positive (W+) An. melas (blue circles) and An. gambiae s.s.-melas hybrids (blue and red triangles). The tree with the highest log likelihood (-1360.58) is shown. The tree is drawn to scale, with branch lengths measured in the number of substitutions per site. The analysis involved 149 nucleotide sequences. There was a total of 528 positions in the final dataset. Relevant subtrees are compressed, labelled with the species and the number of sequences included within them shown in brackets. Accession numbers are shown for sequences obtained from GenBank for comparison, where they are not contained within a subtree for clear visualisation.

Figure 2. An. gambiae complex species phylogenetic analysis of the mitochondrial COI and COII genes. a) Maximum Likelihood molecular phylogenetic analysis of the COI gene for the An. gambiae complex. The tree with the highest log likelihood (-2546.33) is shown. The analysis involved 233 nucleotide sequences. There was a total of 658 positions in the final dataset. b) Maximum Likelihood molecular phylogenetic analysis of the COII gene for the An. gambiae complex. The tree with the highest log likelihood (-2013.22) is shown. The analysis involved 144 nucleotide sequences. There was a total of 728 positions in the final dataset. In both trees the branches where An. gambiae s.s., An. coluzzii and An. arabiensis are grouping are shown in orange, with branches where An. melas, An. merus and An. quadriannulatus are grouping shown in dark blue. Sequences generated in this study are shown in bold, with red for An. gambiae s.s., maroon for An. coluzzii and blue for $A n$. melas sequences. Hybrid samples are shown with triangle node markers, whereas non-hybrids shown with circles. Wolbachia negative specimens are shown with hollow node markers and (W-) following the sample ID, whereas Wolbachia positives are denoted with filled node markers and $(\mathrm{W}+)$ following sample IDs. Accession numbers are shown for sequences obtained from GenBank for comparison, except where duplicate identical sequences were replaced with a single representative, or where subtrees are compressed, for better visualisation. One representative sequence is shown for identical duplicate sequences from the same species, labelled with the species and number of duplicate sequences in brackets, with a diamond node marker. Appropriate subtrees are compressed, labelled with the species included and the number of sequences from each respective species shown in brackets. The trees are drawn to scale, with branch lengths measured in the number of substitutions per site.

Figure 3. An. gambiae complex species phylogenetic analysis of mitochondrial ND4-ND5 gene fragment. a) Overview of the Maximum Likelihood molecular phylogenetic analysis of the ND4-ND5 gene fragment with a subtree compressed in orange where sequences are grouping for An. gambiae s.s., An. coluzzii and An. arabiensis (subtree expanded in b) and a subtree compressed in dark blue where sequences for An. melas, An. merus and An. quadriannulatus are grouping (subtree expanded in c). The tree with the highest log likelihood (-8134.49) is shown. The analysis involved 591 nucleotide sequences. There was a total of 1579 positions in the final dataset. Sequences generated in this study are shown in bold, with red for $A n$ gambiae s.s., maroon for $A n$. coluzzii and blue for $A n$. melas sequences. Hybrid samples are shown with triangle node markers, whereas non-hybrids are shown with circles. Wolbachia negative (W-) specimens are shown with hollow node markers, whereas Wolbachia positives $(\mathrm{W}+)$ are denoted with filled node markers. Accession numbers are shown for sequences obtained from GenBank for comparison, except where duplicate identical sequences were replaced with a single representative, or where subtrees are compressed, for better visualisation. One representative sequence is shown for identical duplicate sequences from the same species, labelled with the species and number of duplicate sequences in brackets, with a diamond node marker. Appropriate subtrees are compressed, labelled with the species included and the number of sequences from each respective species shown in brackets. The tree is drawn to scale, with branch lengths measured in the number of substitutions per site. In c, square brackets denote the grouping of the Western Africa An. melas populations, where sequences generated in this study are also situated, with the Southern Africa and Equatorial Guinea (Bioko Island) populations clustering separately as previously found[61].

Figure 4. Phylogenetic analysis of the An. sp. X ribosomal ITS2, mitochondrial COI and ND4-ND5 gene sequences within the Cellia Subgenus of Anopheles. a) Maximum Likelihood molecular phylogenetic analysis of ribosomal ITS2 sequences. All available GenBank sequences covering the sequenced fragment from the Myzomyia, Neomyzomyia and Annularis series were included, in addition to representative sequences from the An. gambiae complex (Pyretophorus Series) for broader placement and comparison with the other phylogenetic analyses. The tree with the highest log likelihood (-21743.86) is shown. The analysis involved 440 nucleotide sequences. There was a total of 1112 positions in the final dataset. b) Maximum Likelihood molecular phylogenetic analysis of mitochondrial COI sequences. All available GenBank sequences covering the sequenced fragment from the Cellia Subgenus were included. The tree with the highest log likelihood (- 
bioRxiv preprint doi: https://doi.org/10.1101/772855; this version posted November 17,2020 . The copyright holder for this preprint (which was not certified by peer review) is the author/funder, who has granted bioRxiv a license to display the preprint in perpetuity. It is made available under aCC-BY-NC-ND 4.0 International license.

7291.31 ) is shown. The analysis involved 157 nucleotide sequences. There was a total of 658 positions in the final dataset. c) Maximum Likelihood molecular phylogenetic analysis of mitochondrial ND4-ND5 sequences. All available GenBank sequences covering the sequenced fragment from the Cellia Subgenus were included. The tree with the highest log likelihood (-11183.21) is shown. The analysis involved 95 nucleotide sequences. There was a total of 1518 positions in the final dataset. In all trees the sequences generated in this study are shown in bold, with the Wolbachia positive An. sp. X specimen (SENP2.E2 $(\mathrm{W}+)$ ) shown in green, with a filled circle node marker. Branches where sequences from the $M y z o m y i a$ series are grouping are shown in dark green, and with an external labelled dark green bracket to denote this grouping. Relevant subtrees are compressed, labelled with the species and the number of sequences included within them shown in brackets. Accession numbers are shown for sequences obtained from GenBank for comparison, where they are not contained within a subtree for clear visualisation.

Figure 5. Wolbachia strain phylogenetic analysis using the $16 S$ r RNA gene. Maximum Likelihood molecular phylogenetic analysis of the 16S rRNA gene for Wolbachia strains detected in Anopheles specimens from Guinea. The tree with the highest $\log$ likelihood (-1013.48) is shown. The tree is drawn to scale, with branch lengths measured in the number of substitutions per site. The analysis involved 34 nucleotide sequences. There was a total of 398 positions in the final dataset. Sequences generated in this study are shown in bold with filled circle node markers. The wAnga-Guinea sequences from both A and B Supergroups are shown in purple. The $w$ Ans $X$ strain is shown in green and the $w$ Wat strain obtained from $C x$. watti for comparative work is shown in light blue. wAnga sequences obtained from the An. gambiae complex species previously are shown in orange with their accession numbers. Wolbachia strains obtained from other mosquito species in previous studies are shown in navy blue with their accession numbers. Additional Wolbachia sequences from non-mosquito hosts obtained from GenBank for comparison are shown with their accession numbers, in black.

Figure 6. Wolbachia strain phylogenetic analysis using the wsp gene. Maximum Likelihood molecular phylogenetic analysis of the wsp gene for resident strains in Anopheles species from Guinea. The tree with the highest log likelihood (-3646.57) is shown. The tree is drawn to scale, with branch lengths measured in the number of substitutions per site. The analysis involved 86 nucleotide sequences. There was a total of 431 positions in the final dataset. Sequences generated in this study are shown in bold with filled circle node markers. wAnga-Guinea-A1 wsp from an An. melas specimen is shown in purple, and $w$ Ans X is shown in green. Reference numbers of additional sequences obtained from the MLST database (IsoN; Isolate number) or GenBank (accession number) are shown. Strains isolated from mosquitoes are shown in blue, with those strains from other Anopheles species highlighted in bold.

Figure 7. Wolbachia multilocus sequence typing (MLST) phylogenetic analysis of $w$ Anga-Guinea-A1 and $w$ AnsX. Maximum Likelihood molecular phylogenetic analysis from concatenation of all five MLST gene loci for resident Wolbachia strains wAnga-Guinea-A1 (in purple) and wAnsX (in green). Concatenated sequences obtained in this study are highlighted in bold with a filled circle node marker. The tree with the highest log likelihood (-11404.41) is shown and drawn to scale, with branch lengths measured in the number of substitutions per site. The analysis involved 102 nucleotide sequences. There were a total of 2063 positions in the final dataset. The concatenated MLST sequence data from wAnga-Ghana obtained from An. coluzzii in a previous study[26] is shown in orange. Concatenated sequence data from Wolbachia strains downloaded from the MLST database for comparison are shown with isolate numbers in brackets (IsoN). Wolbachia strains isolated from mosquito species are shown in blue, with those strains from other Anopheles species highlighted in bold. Strains isolated from other Dipteran species are shown in navy blue, from Coleoptera in olive green, from Hemiptera in purple, from Hymenoptera in teal blue, from Lepidoptera in maroon and from other, or unknown orders in black.

Figure 8. Wolbachia strain densities in wild-caught female mosquitoes from the Maferinyah sub-prefecture of Guinea. Total RNA extracted from individual mosquitoes was standardised to $2.0 \mathrm{ng} / \mu \mathrm{L}$ prior to being used in qRT-PCR assays targeting the Wolbachia $16 S r R N A$ gene. A synthetic oligonucleotide standard was designed to calculate $16 S$ $r R N A$ gene copies per $\mu \mathrm{L}$ of RNA using a serial dilution series and all samples were run in triplicate in addition to no template controls.

Table 1. Wolbachia prevalence rates in Anopheles species collected in two regions of Guinea in 2018. Species containing Wolbachia-infected individuals are denoted in bold.

Table 2. Novel resident Wolbachia strain WSP typing and multilocus sequence typing (MLST) gene allelic profiles. Newly assigned novel alleles for $w$ Ans $X$ are shown in bold red font. ${ }^{*} w$ Anga-Guinea-A1 hcp $A$ could not be assigned a novel allele number due to a possible double infection which was unresolvable, therefore the allele number of the closest match $(\mathrm{CM})$ is shown with the number of single nucleotide differences to the closest match in brackets. 


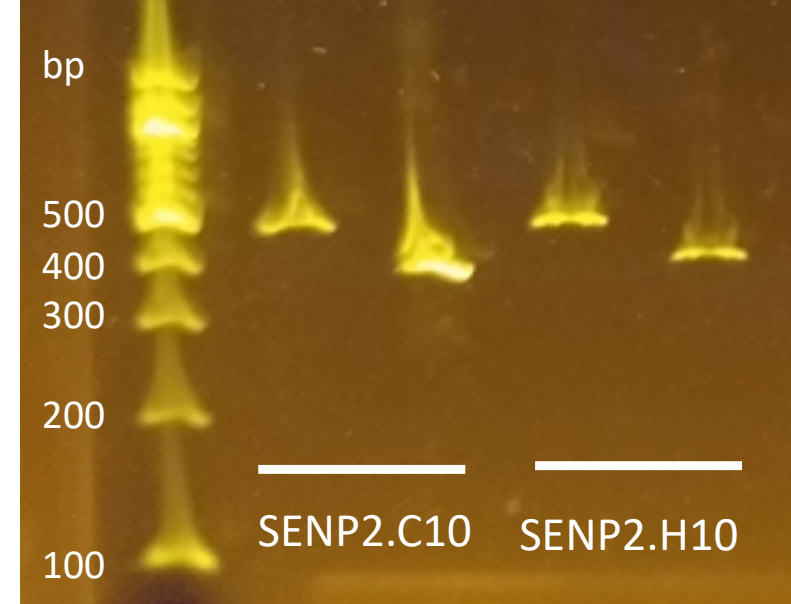

$61=$ An. gambiae (10)

MH598415.1 An. gambiae GUI-KSK2

(1)

63. An. gambiae (3)

(1)

MH598438. 1 An. gambiae UGA-BUT3

MH598439.1 An O

EU104634. A An. gambiae 804 (M form - coluzzii)

EU104635.1 An. gambiae 900 (M form - coluzzii)

54 EU4104636.1 An. gambiae 8558 (M form - coluzzii)

EU104637. 1 An. gambiae 8607 (M form - coluzzii)

EU104638.1 An. gambiae 8610 (M form - coluzzii)

EU104639.1 An. gambiae H39 (M form - coluzzii)

EU104640.1 An. gambiae 8432 (M form - coluzzii)

EU104641.1 An. gambiae 8600 (M form - coluzzii)

EU104642.1 An. gambiae 8606 ( $M$ form - coluzzii)

EU104643.1 An. gambiae 8642 (M form - coluzzii)

AF470116.1 An gambiae ss LD10

80 AF470115.1 An gambiae ss Boh27

b

98

A SENP2.C10 (hybrid - gambiae/coluzzii primers) (W+)

GQ870317.1 An. gambiae 104

KT160243.1 An. gambiae Lofa1

$\triangle \mathrm{SENP2.C10( \textrm {W } + )}$

GQ870319.1 An. gambiae 220

A SENP2.H12(W+)

KT160244.1 An. coluzzii Kara2

JN994138.1 An. gambiae

GQ870315.1 An. gambiae 10573

MH598434.1 An. coluzzil GHA-DOG5

MH598433. 1 An. coluzziI GHA-DOG4

MH598431. 1 An. coluzzIII GHA-DOG2

MH598430. 1 An. coluzzii GHA-DOG

55 SENP2.H10 (hybrid - gambiae/coluzzii primers) (W+)

AF470112.1 An coluzzii (gambiae M form) Boh33

- AF470111.1 An arabiensis Tsa9

U10138.1 An arabiensis ARZAG

$100-$ AF470095.1 An arabiensis Armor3

AF470110.1 An arabiensis Tsa5

U10137.1 An quadriannulatus CHIL

67

AF438154.1 An quadriannulatus B

84 AF438153.1 An quadriannulatus B

AH006430.1 An merus V12

A SENP2.H10 (hybrid - melas primers) (W+)

AH006429.1 An melas BAL

99 SENP2.C10 (hybrid - melas primers) (W+)

- DQ385830.1 An bwambae AnoB1

65 DQ385831.1 An bwambae Bb50

GQ870313.1 An. merus 10304

SENP2

MH598435. 1 An melas GH

GQ870314 1 An melas GHA-DOGO

SENP2.B9 $(\mathrm{W}+)$

SENP2.H9 $(\mathrm{W}+)$

A SENP2.H10 (W-

SENP2.A12 $(\mathrm{W}+)$
SENP2.G2 $(\mathrm{W}+)$ 
not certified by peer review) is the author/funder, who has granted bioRxiv a license to display the preprint in perpetuity. It is made availa under aCC-BY-NC-ND 4.0 International license.

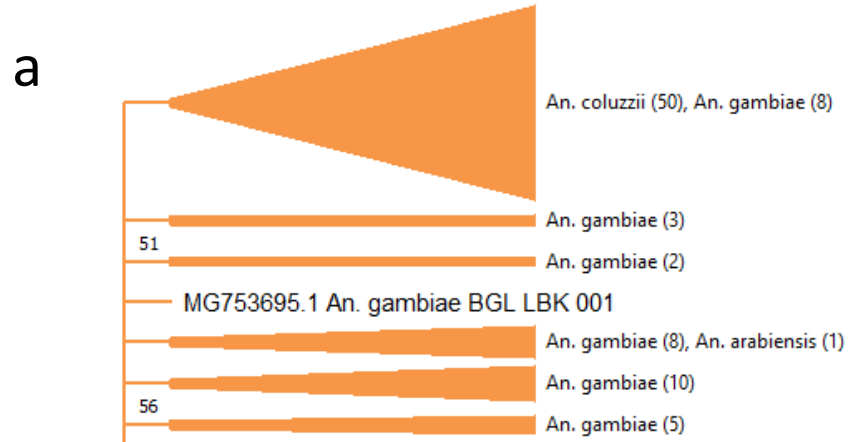

_ MG930831.1 An. gambiae 2011MUTE483

An. gambiae (2)
An. gambiae (6)
An. gambiae (2)
An. gambiae (6)
An. gambiae (3)
An. gambiae (2)

${ }^{65}$ DQ792576.1 An. arabiensis EM1

— MG753750.1 An. gambiae SYR 008

${ }_{64}$ MG753696.1 An. gambiae BGL LBK 002

— MG753761.1 An. gambiae WML 001

$\checkmark$ An. gambiae (39)

KY670610.1 An. arabiensis

KM068069.1 An. arabiensis AraJZ19

- MG930848.1 An. gambiae MINE105

_ MG930842.1 An. gambiae LUPI074
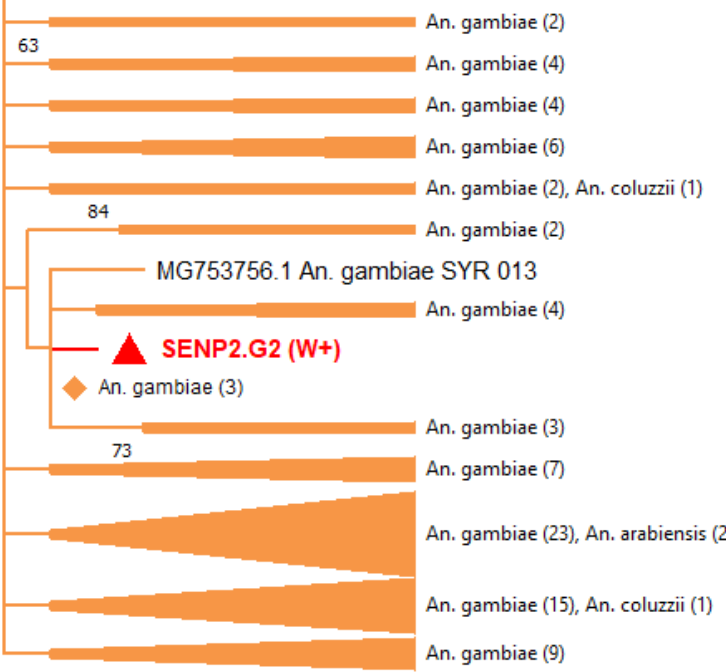

MG930835.1 An. gambiae 2011MUTE493

An. gambiae (2)

- MG930891.1 An. gambiae 2012KELA321

MG930852.1 An. gambiae OKJ045

KR152319.1 An. coluzzii Grandbassa3

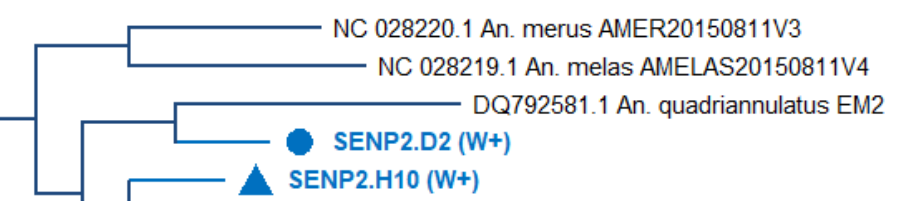

89 SENP2.B9 (W+)

DQ792579.1 An. melas JA22

DQ792580.1 An. melas EM5

$\triangle$ SENP2.C10 $(\mathrm{W}+)$

SENP2.A10 (W+)

SENP2.C12 $(\mathrm{W}+)$

SENP2.A12 $(\mathrm{W}+)$

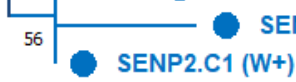

0.0050

b MG930877.1 An. gambiae 2012KELA240

60 MG753711.1 An. gambiae BWM 006

$\triangle$ FANP2.G4 (W-)

- MG930890.1 An. gambiae 2012KELA285

- MG930876.1 An. gambiae 2012KELA239

$\checkmark$ An. gambiae (51)

NC 028212.1 An. arabiensis AARAB20150811V4

An. gambiae (3)

An. gambiae (3)

An. gambiae (4)

$\triangle$ FANP2.H2 (W-)

MG753697.1 An. gambiae BGL LBK 003 MG753717.1 An. gambiae KZI 013

An. gambiae (2)

MG753659.1 An. gambiae BDA 003

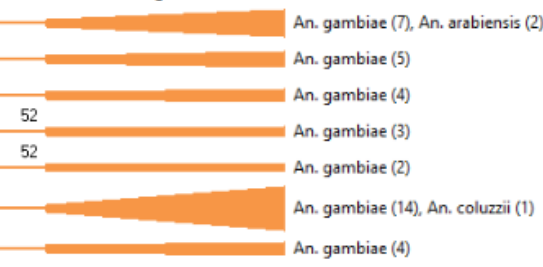

$\frac{60}{62}$ DQ792577.1 An. gambiae EM3

An. gambiae (33) An. gambiae (3)

$\triangle$ SENP1.E11 (W-)

\begin{tabular}{r}
\hline $52 \Delta$ An. gambiae (5) \\
An. gambiae (4) \\
An. gambiae (4)
\end{tabular}

MG930874.1 An. gambiae 2012KELA214

- MG753765.1 An. gambiae WML 005

- An. gambiae (14)

MK755601.1 An coluzzii LOP13

NC 028215.1 An. coluzzii ACOLU20150811V3

$\triangle$ FANP2.F4 (W-)

$\triangle$ MAFP1.B9 (W-)

O MAFP1.C8 (W-)

An. gambiae (3)

MG753720.1 An. gambiae KZI 004

58 A SENP2.G2 $(\mathrm{W}+)$

MG753750.1 An. gambiae SYR 008

- MG930881.1 An. gambiae 2012KELA443

- DQ792581.1 An. quadriannulatus EM2

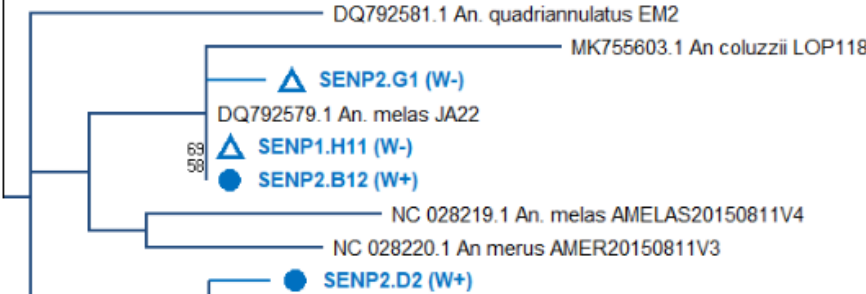

O SENP2.F2 (W-)

A SENP2.H10 (W+)

SENP2.H11 (W+)

DQ792580.1 An. melas EM5

A SENP2.G10(W+)

$\triangle$ SENP2.G12 (W+)

SENP2.G12 (W+)
SENP2.A12 (W+)

- SENP2.B1 (W+)

- SENP2.C2 $(W+)$

SENP2.G11 (W+) O SENP1.F10 (W-

SENP2.B10 $(W+)$

SENP2.A10 (W+)

SENP2.C1 $(W+)$

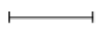



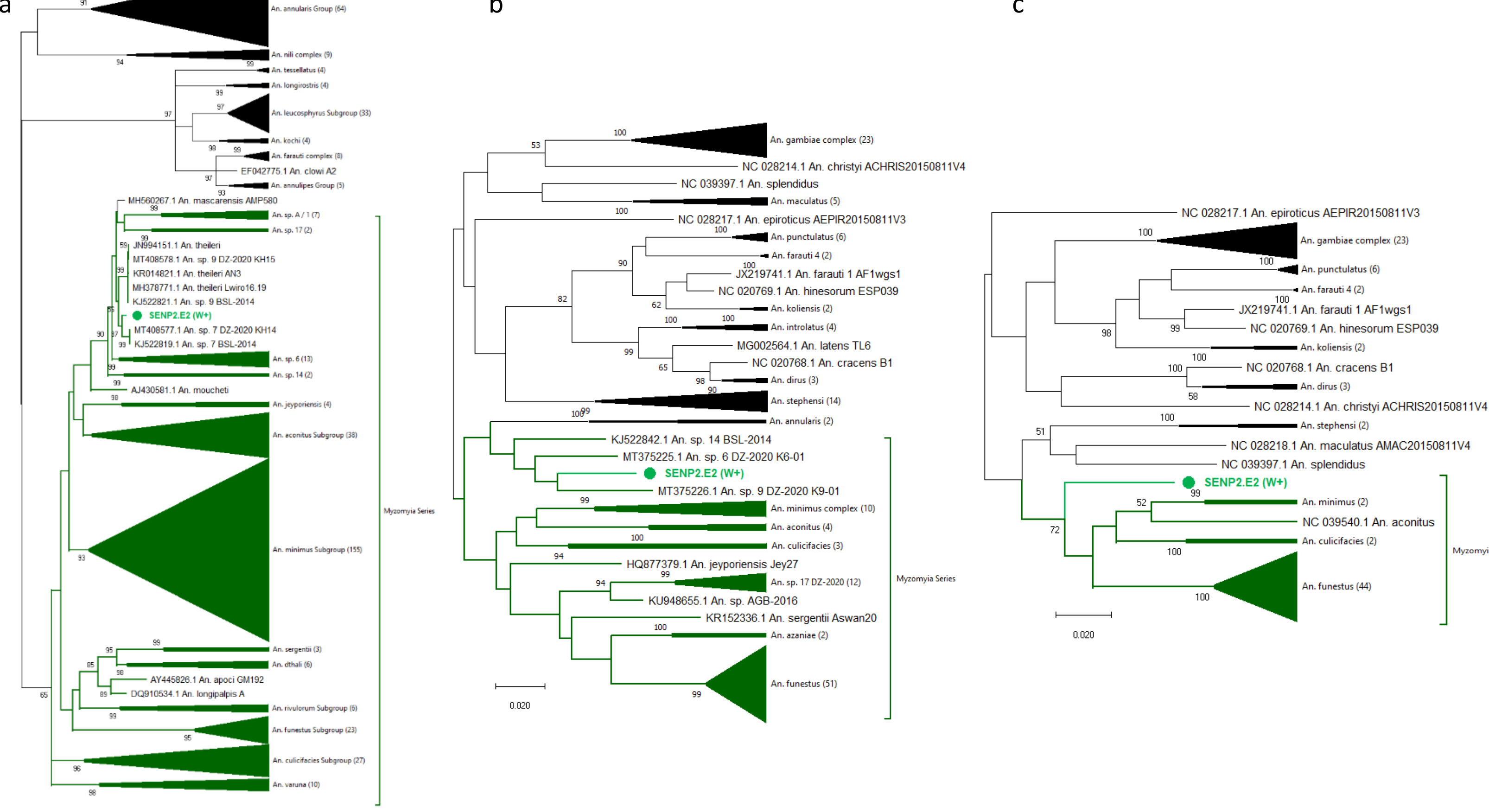

0.020 


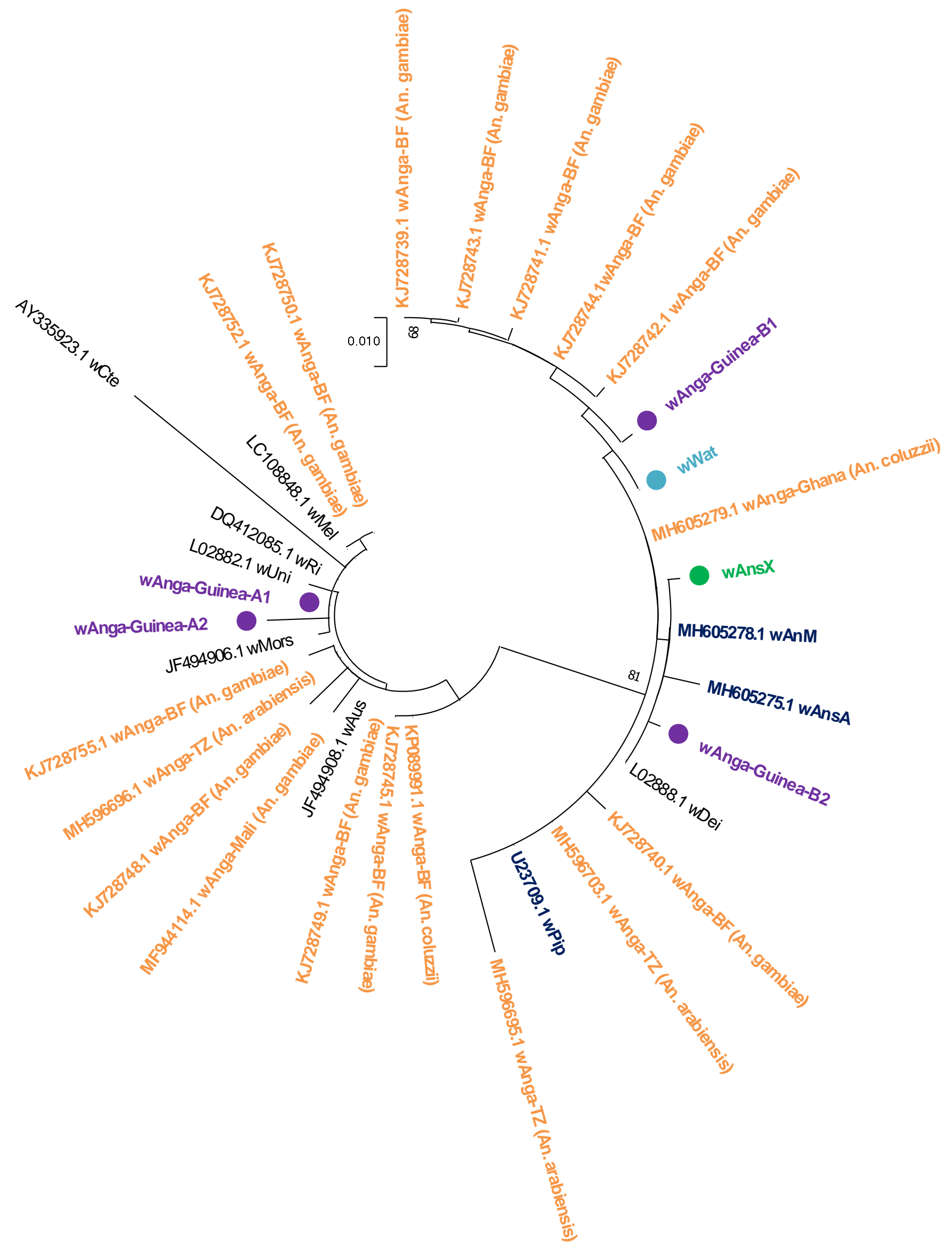




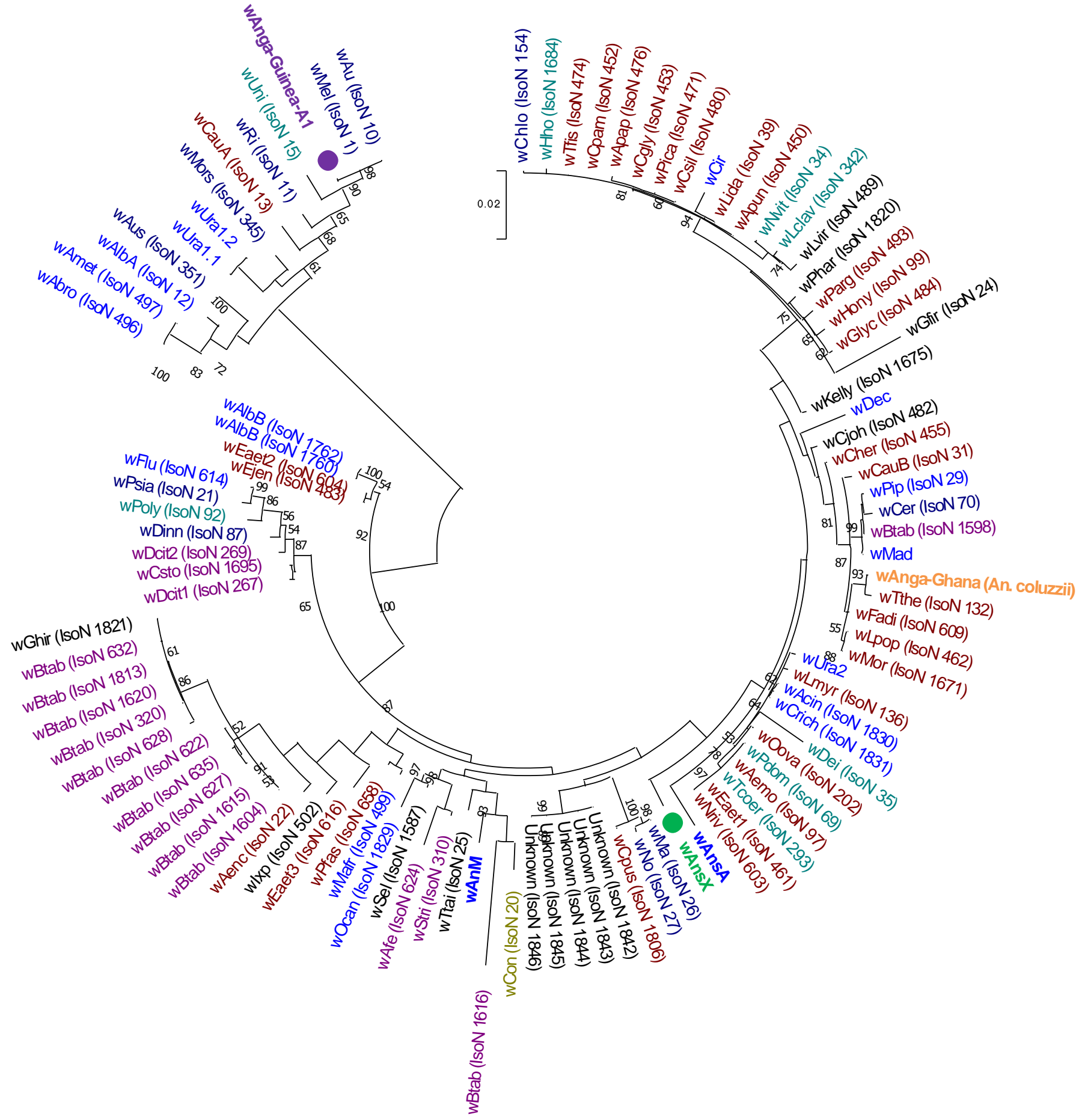




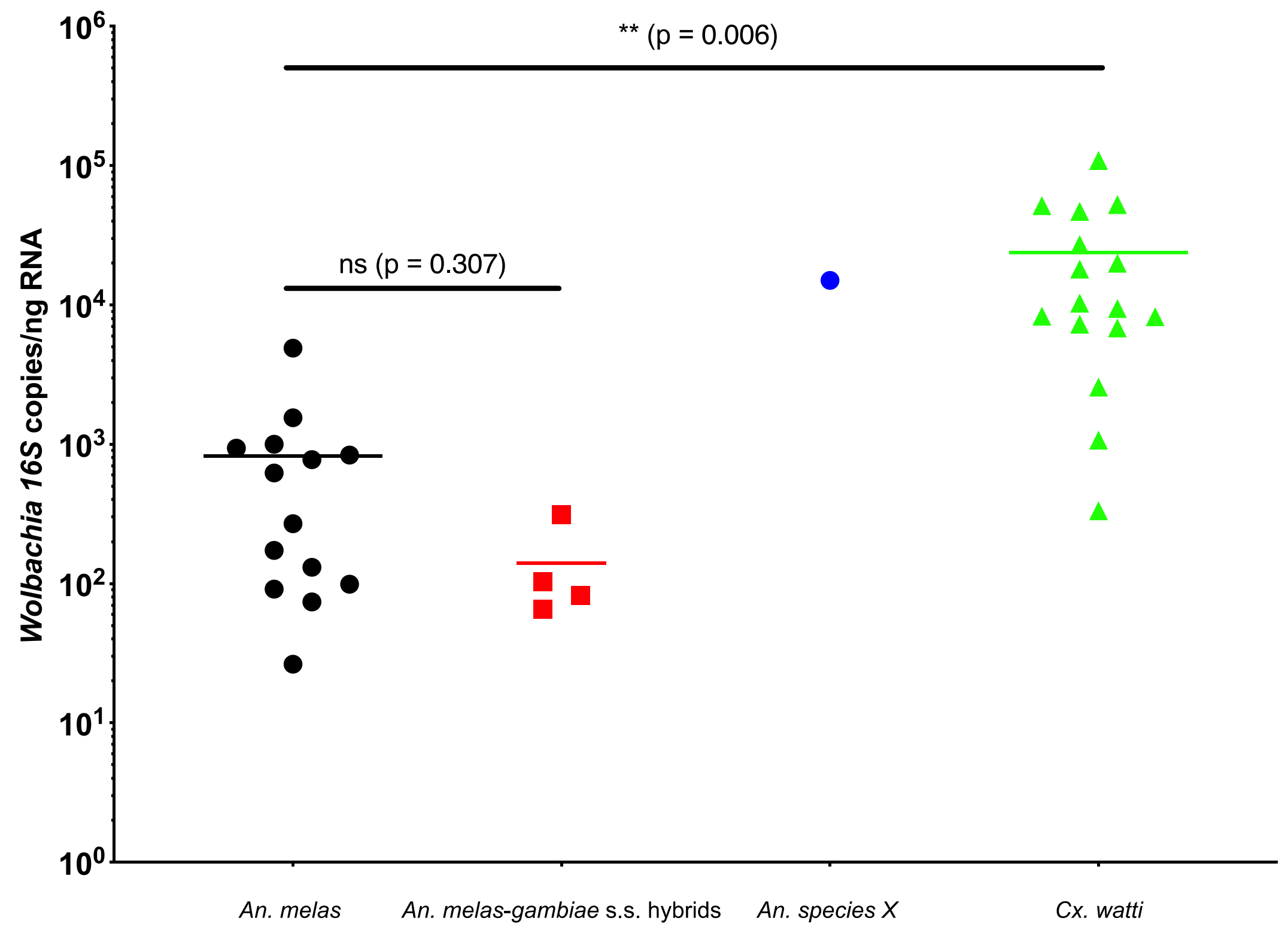

